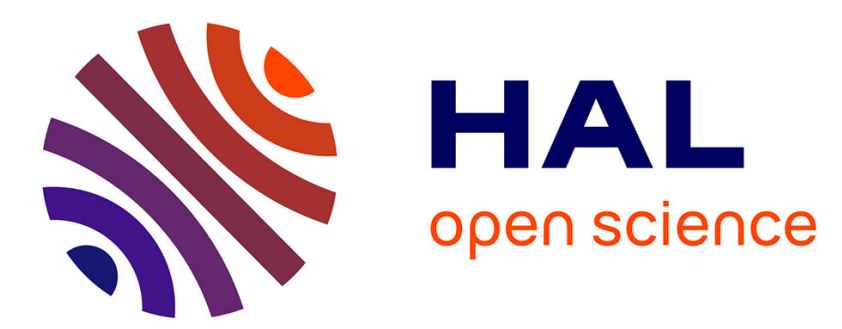

\title{
Effervescence in champagne and sparkling wines: From bubble bursting to droplet evaporation
}

Thomas Séon, Gérard Liger-Belair

\section{To cite this version:}

Thomas Séon, Gérard Liger-Belair. Effervescence in champagne and sparkling wines: From bubble bursting to droplet evaporation. The European Physical Journal. Special Topics, 2017, 226, pp.117156. 10.1140/epjst/e2017-02679-6 . hal-01447495

\section{HAL Id: hal-01447495 \\ https://hal.science/hal-01447495}

Submitted on 26 Jan 2017

HAL is a multi-disciplinary open access archive for the deposit and dissemination of scientific research documents, whether they are published or not. The documents may come from teaching and research institutions in France or abroad, or from public or private research centers.
L'archive ouverte pluridisciplinaire HAL, est destinée au dépôt et à la diffusion de documents scientifiques de niveau recherche, publiés ou non, émanant des établissements d'enseignement et de recherche français ou étrangers, des laboratoires publics ou privés. 


\title{
Effervescence in Champagne and Sparkling Wines
}

\section{From bubble bursting to drop evaporation}

T. Séon ${ }^{1, a}$ and G. Liger-Belair ${ }^{2}$

1 Sorbonne Universités, Université Pierre et Marie Curie, and Centre National de la Recherche Scientifique, Unité Mixte de Recherche 7190, Institut Jean Le Rond d'Alembert, 4 Place Jussieu, F-75005 Paris, France.

2 Équipe Effervescence (GSMA), UMR CNRS 7331, Université de Reims Champagne-Ardenne, BP 1039, 51687 Reims, France.

\begin{abstract}
When a bubble reaches an air-liquid interface, it ruptures, projecting a multitude of tiny droplets in the air. Across the oceans, an estimated $10^{18}$ to $10^{20}$ bubbles burst every second, and form the so called sea spray, a major player in earth's climate system. At a smaller scale, in a glass of champagne about a million bubbles nucleate on the wall, rise towards the surface and burst, giving birth to a particular aerosol that holds a concentrate of wine aromas. Based on the model experiment of a single bubble bursting in simple liquids, we depict each step of this effervescence, from bubble bursting to drop evaporation. In particular, we propose simple scaling laws for the jet velocity and the top drop size. We unravel experimentally the intricate roles of bubble shape, capillary waves, gravity, and liquid properties in the jet dynamics and the drop detachment. We demonstrate how damping action of viscosity produces faster and smaller droplets and more generally how liquid properties enable to control the bubble bursting aerosol characteristics. In this context, the particular case of Champagne wine aerosol is studied in details and the key features of this aerosol are identified. We demonstrate that compared to a still wine, champagne fizz drastically enhances the transfer of liquid into the atmosphere. Conditions on bubble radius and wine viscosity that optimize aerosol evaporation are provided. These results pave the way towards the fine tuning of aerosol characteristics and flavor release during sparkling wine tasting, a major issue of the sparkling wine industry.
\end{abstract}

\section{Introduction}

\subsection{Bubble bursting and effervescence}

As champagne or sparkling wine is poured into a glass, the myriad of ascending bubbles collapse and therefore radiate a multitude of tiny droplets above the free

\footnotetext{
${ }^{a}$ e-mail: thomas.seon@gmail.com
} 

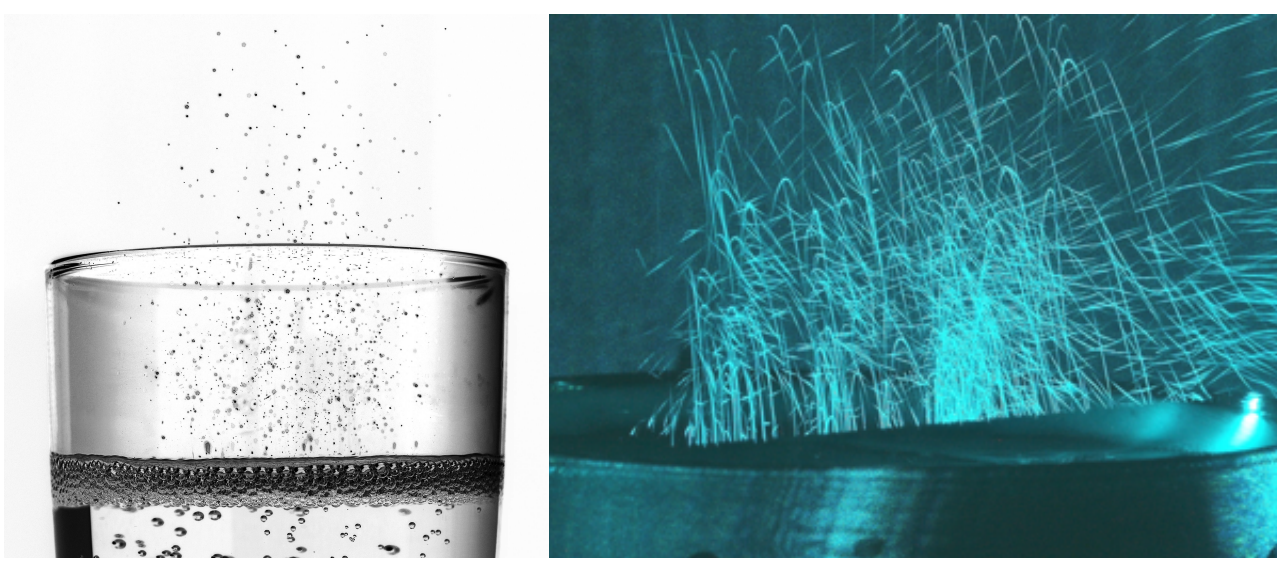

Fig. 1. Image on the left : the collapse of hundreds of bubbles at the free surface radiate a cloud of tiny droplets which is characteristic of champagne and other sparkling wines and which complements the sensual experience of the taster (C)Alain Cornu / Collection CIVC). On the right : The aerosol constituted by myriads of tiny droplets ejected from bubbles' bursting above the surface of a coupe, as seen through laser tomography technique; the droplets' trajectories are materialised by blue streaks of light during the 1s-exposure time of a digital photo camera. (Photograph by G. Liger-Belair, F. Beaumont and G. Polidori.)

surface, into the form of very characteristic and refreshing aerosols, as shown in the photograph displayed on the left of Fig. 1. On the image on the right, laser tomography techniques were applied to freeze this huge number of bursting events and all the droplets ejected above champagne glasses in real consuming conditions ${ }^{1}$.

Indeed, it is now generally recognized that bubbles bursting at a liquid surface eject two kinds of droplets (see Fig. 2): (i) small droplets, called film drops, formed as the film of the emerged bubble-cap disintegrates ${ }^{2 ; 3 ; 4}$, and (ii) bigger jet drops, formed as the so-called "Worthington jet", driven by the collapse of the unstable immersed cavity $^{5 ; 6 ; 7}$, ruptures through end-pinching mechanism ${ }^{8 ; 9 ; 10}$

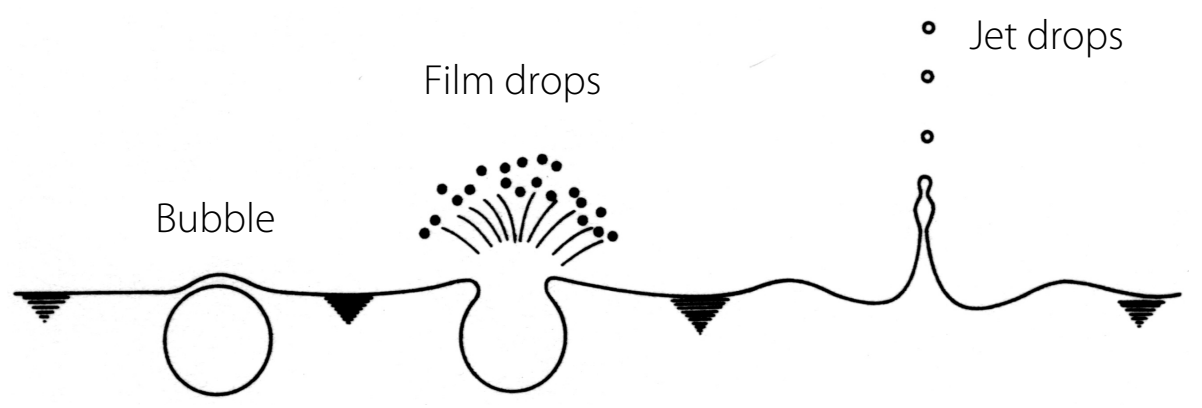

Fig. 2. Scheme of the two production ways of droplets from a bursting bubble; redrawn from the article by Resch et al. ${ }^{11}$.

On a larger scale, sea spray, known to transports dissolved gases, salts, surfactants, and biological materials to the atmosphere, is largely attributed to aerosols produced by an estimated $10^{18}$ to $10^{20}$ bubbles that burst every second across the oceans ${ }^{12 ; 13 ; 14 ; 15 ; 16 ; 17}$. This marine aerosols indeed account for the majority of the 
global natural aerosol flux, thereby having a significant impact the Earth's climate system and biogeochemical cycling ${ }^{18 ; 17}$.

\subsection{Champagne fizz : a concentrate of champagne aromas}

Marine aerosols have also been well described because of their important role in the sea-air exchange of surfactant materials ${ }^{19 ; 20 ; 21}$. Indeed, as a bubble rises in the ocean, the amphiphilic properties of surfactants lead them to its surface. Therefore, bubbles drag surfactants all along their way through the liquid bulk, reach the sea surface, to finally burst. The sea spray aerosol there produced is then highly enriched with these surface active agents. Consequently, during rough sea conditions, when a large amount of bubbles are entrapped, surfactant concentrations in aerosols are found to be increased by several orders of magnitude compared with those found in the liquid bulk ${ }^{17}$.

From a conceptual point of view, the situation found in glasses of champagne, sparkling wines, and fizzy beverages in general, is finally very similar. Champagne holds indeed hundreds of surface active compounds. Once champagne is poured into a glass, bubbles nucleated on the glass wall and champagne surfactants are then dragged along with ascending bubbles through the liquid bulk ${ }^{22}$. Surfactants finally reach the free surface and concentrate themselves at the air/champagne interface. Note that one of the consequences of the increasing concentration of surfactants at the champagne surface is the increasing lifetime of bubbles with time ${ }^{23 ; 24}$ that can be observed during tasting.

A few years ago, ultrahigh resolution mass spectrometry was used in order to analyze the droplets released by bubbles bursting in champagne ${ }^{25}$. It was found that this aerosol was indeed considerably enriched, compared with the champagne bulk, with chemical compounds showing both surface activity and organoleptic interest. This signifies that the aerosol found in the headspace above a glass poured with champagne, the so-called champagne fizz, actually holds the organoleptic "essence" of champagne. In other words, those droplets carry a concentrate of champagne aromas. While flying above the glass they are expected to partly evaporate, accelerating the transfer of the numerous aromatic volatile organic compounds above the liquid surface $^{26}$. This recent discovery supports the idea that rising and collapsing bubbles act as a continuous paternoster lift for aromas above every glass of champagne or any flavored carbonated beverage.

In the following, our objectives are both to improve our understanding of the effervescence physics, from bubble bursting to drop evaporation and to study, in the context of champagne, the aerosol evaporation as a function of the effervescence control parameters in order to optimize the aroma diffusion.

\section{Experimental context}

We start by looking at the specificity of bubble bursting in champagne. This will allow us to precise the outline of the experimental study.

\subsection{Bubble bursting in champagne}

\subsubsection{Film drops}

As introduced above, bursting bubble usually leads to drop production through two different mechanisms : film drops through film retraction and jet drops when jet ruptures. Figure 3 (a) shows a sequence of a bubble lying at the free surface of water, 
(a) Water

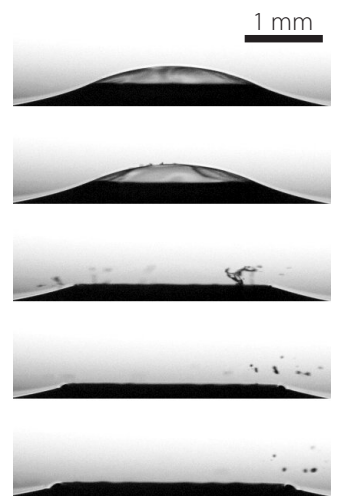

(b) Champagne

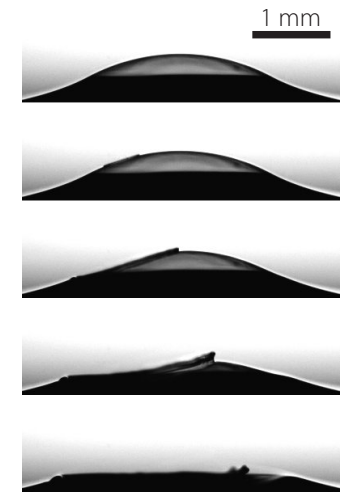

(c) Water-Ethanol Solution

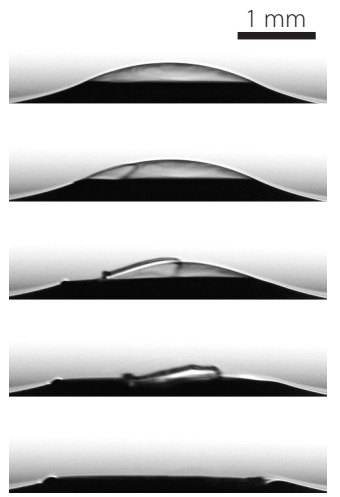

Fig. 3. Experimental time sequences of a bubble of radius $R_{b}=1.7 \mathrm{~mm}$ bursting in : (a) demineralized water, (b) champagne at $20^{\circ} \mathrm{C}$ and (c) a water-ethanol solution with the same properties. Liquid properties are the same as in Fig. 4. Space scale is on each sequence. The time between each image is : (a) $\Delta \mathrm{t}=77 \mu \mathrm{s}$, (b) $\Delta \mathrm{t}=83 \mu \mathrm{s}$ and (c) $\Delta \mathrm{t}=77 \mu \mathrm{s}$.

after a few seconds the cap film punctures and retracts. During retraction the rim suffers an inertial destabilization of a Rayleigh-Taylor type, which leads to the formation of ligaments. The ligaments are then stretched out by centrifugation, producing disjointed droplets by a Plateau-Rayleigh destabilization ${ }^{4}$. These are the so-called film drops that we observe on the two last pictures of the sequence (a). In water and for the bubble radius considered here $\left(R_{b}=1.7 \mathrm{~mm}\right)$ film rupture should generate thirty-four drops of average radius seventeen microns ${ }^{4}$. But this applies to water. In particular, Fig. 3 (b) presents the same film retraction sequence in champagne, and in this case we observe no film drops. The retraction velocity appears lower in champagne suggesting that destabilization of the rim cannot develop before the film has disappeared ${ }^{27}$.

This unexpected result might have various causes as champagne is different from water in many aspects. It is first more viscous (1.6 mPa.s at room temperature) and has a lower surface tension $\left(48 \mathrm{mN} \cdot \mathrm{m}^{-1}\right)$. Gradients of surface tension, inherent to hydro-alcoholic solution ${ }^{28}$, can lead to a film thickening through Marangoni effect before it bursts. All these effects work toward the stabilization of the film retraction and, therefore, the film drops removal, however we do not know which one prevails. The exact reason of this disappearance of film drops definitively constitutes a fascinating problem which needs be addressed. Anyway, it makes champagne aerosol very different from sea spray and an interesting model aerosol only populated by jet drops.

\subsubsection{Jet drops}

Figure 4 (a) displays a sequence of a typical jetting event following a bubble bursting at the free surface of a Champagne wine. The first image shows the static bubble, then the film separating the bubble from the atmosphere drains and bursts leaving an unstable open cavity. This (sub)millimetric cavity relaxes due to capillary forces and gives rise to the high speed vertical jet shooting out above the free surface as observed on the sequence. The jet then fragments into droplets, generating an aerosol of a few jet drops, the only constituent of a champagne aerosol. 


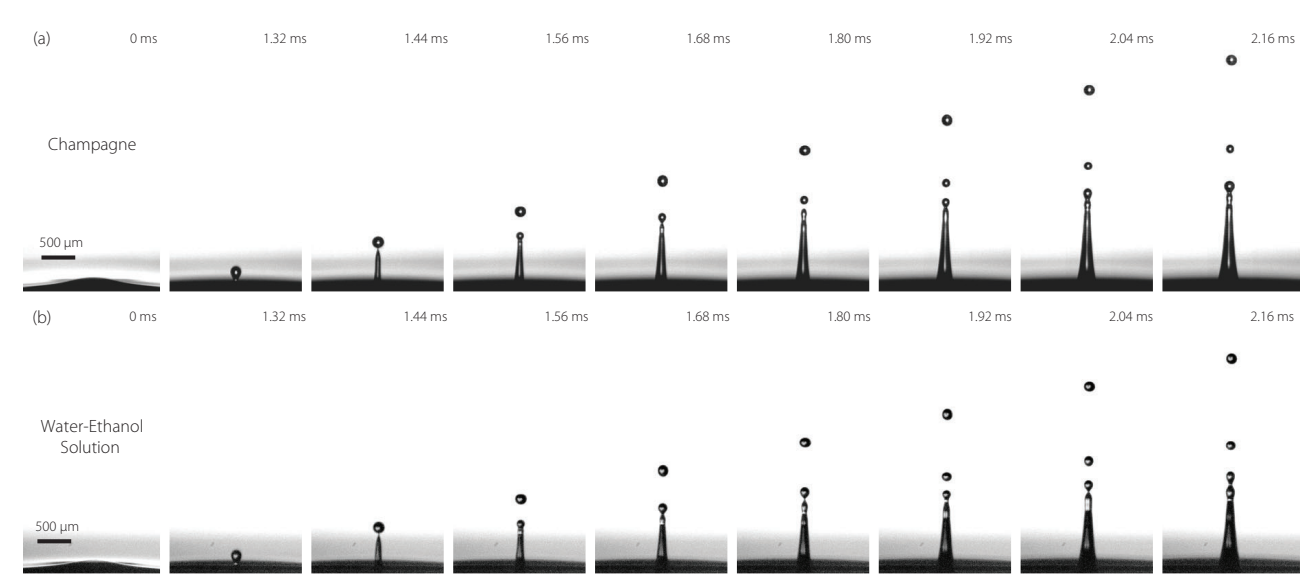

Fig. 4. Experimental time sequences of the typical jetting events following a bubble bursting at a free surface of (a) a Champagne wine at $20^{\circ} \mathrm{C}$ (viscosity $\mu=1.6 \mathrm{mPa}$.s, surface tension $\gamma=48 \mathrm{mN} \cdot \mathrm{m}^{-1}$ and density $\rho=992 \mathrm{~kg} \cdot \mathrm{m}^{-3}$ ) and (b) a water-ethanol solution (resp. 89,5 $\%$ and $10,5 \%$ of total weight) with the same properties. Space scale is on each sequence and times are shown on the snapshots. Bubble radius $R_{b}$ is almost the same for the two sequences : (a) $R_{b}=817 \mu \mathrm{m}$ and (b) $R_{b}=830 \mu \mathrm{m}$.

\subsubsection{Idealized Champagne wine}

In the aim of establishing an idealized champagne for our study, the same bursting bubble experiment has been realized in a simple water-ethanol solution (resp. 89,5\% and $10,5 \%$ of total weight), allowing us to mimic the liquid properties of a standard Champagne wine at $20^{\circ} \mathrm{C}$ : viscosity $\mu=1.6 \mathrm{mPa}$.s and surface tension $\gamma=48$ $\mathrm{mN} \cdot \mathrm{m}^{-11}$. Figures 4 (b) and 3 (c) respectively present the jetting event and film retraction sequences following a bubble bursting in our hydro-alcoholic solution, for a bubble of the same size as Fig. 4 (a) and 3 (b). The sequences are very close to each other. In particular no film drops are produced and the jet drops size and dynamics look very similar. Therefore, both in terms of the rim retraction and the jet dynamics, a champagne at room temperature is qualitatively perfectly mimicked by a water-ethanol solution with the same properties. As a consequence, the role of surfactants - always present in champagne - seems to be negligible in the bubble bursting dynamics and the drop ejection. These surprising qualitative results will be quantitatively confirmed in the following (Fig. 19).

Champagne is a complex hydro-alcoholic solution holding tens of aromatic compounds, and a small amount of surface-active macromolecules with a concentration of only a few milligrams per liter ${ }^{29}$. Such a small amount of surface-active materials in champagne was actually found to have only little effect on ascending bubble dynamics ${ }^{29}$. It also has almost no effect on equilibrium surface tension as, our hydroalcoholic solution has the same surface tension as a real champagne (see Table 1), with a very close relative amount of ethanol : $13 \%$ of total volume in our solution (which corresponds to $10.5 \%$ in mass) and $12.5 \%$ in champagne. All these arguments support the idea that surfactants do not affect the dynamics of bursting bubbles in a real champagne. Consequently, we believe that it will be reasonable not to take surfactants into account here, in the dynamics and the evaporation of jet droplets above a champagne surface. Nevertheless, it makes no doubt that the fine study of such influence should be carried out in further studies.

Furthermore, champagne is always served at a temperature lower than room temperature, leading to an increase of the liquid viscosity. Glycerol, a water-soluble vis- 


\begin{tabular}{|l|c|c|c|c|}
\hline & $(\mathrm{i})$ & $(\mathrm{ii})$ & $(\mathrm{iii})$ & Champagne $\left(20^{\circ} \mathrm{C}\right)$ \\
\hline Water (\%) & 89.5 & 66.6 & 55.3 & - \\
\hline Ethanol (\%) & 10.5 & 7.6 & 4.9 & - \\
\hline Glycérol $(\%)$ & 0 & 25.8 & 39.8 & - \\
\hline$\mu(\mathrm{mPa} . \mathrm{s})$ & 1.6 & 2.6 & 3.6 & 1.6 \\
\hline$\gamma\left(\mathrm{mN} . \mathrm{m}^{-1}\right)$ & 48 & 47 & 47.5 & 48 \\
\hline$\rho\left(\mathrm{kg} . \mathrm{m}^{3}\right)$ & 983 & 1047 & 1082 & 992 \\
\hline
\end{tabular}

Table 1. Characteristics of the three idealized champagne (i), (ii), (iii) and of champagne at $20^{\circ} \mathrm{C} . \mu, \rho$ and $\gamma$ stand for viscosity (mPa.s), density $\left(\mathrm{kg} . \mathrm{m}^{-3}\right)$ and surface tension $\left(\mathrm{mN}^{-1} \mathrm{~m}^{-1}\right)$.

cous liquid, can therefore be added in the mixtures to tune their viscosity and mimic this temperature effect. Consequently, three mixtures of water, ethanol and glycerol - solutions (i)-(iii) - with $\gamma=48 \mathrm{mN} . \mathrm{m}^{-1}$ and respectively $\mu=1.6,2.6$ and 3.6 m.Pa.s (see Table 1) will be used in order to reproduce the liquid properties of a usual champagne at three different temperatures, namely 20,12 and $4^{\circ} \mathrm{C}^{1}$. These will be our idealized champagne solutions. In addition, degassed champagne at room temperature will also be used in order to compare the results with the water-ethanol solution (i).

\subsection{Experimental setup}

\subsubsection{Most of our liquids are not idealized champagne}

In addition to the idealized champagne introduced in the previous section and used in the particular cases of application to champagne (see Table 1), others solutions with broader parameter ranges are used in order to characterize more generally the different regimes of bubble bursting. These liquids include nine water-glycerol mixtures of viscosity in the range $\mu=1 \mathrm{mPa} . \mathrm{s}-12 \mathrm{mPa} . \mathrm{s}$, surface tension $\gamma=64 \mathrm{mN} . \mathrm{m}^{-1}-72$ $\mathrm{mN} \cdot \mathrm{m}^{-1}$, and density $\rho=1000 \mathrm{~kg} \cdot \mathrm{m}^{-3}-1160 \mathrm{~kg} \cdot \mathrm{m}^{-3}$ and ethanol $(\mu=1.2 \mathrm{mPa} . \mathrm{s}$, $\gamma=23 \mathrm{mN} \cdot \mathrm{m}^{-1}, \rho=780 \mathrm{~kg} \cdot \mathrm{m}^{-3}$ ) (see Table 2 ).

\begin{tabular}{|l|c|c|c|c|c|c|c|c|c|c|}
\hline & Wat. & S2 & S3 & S4 & S5 & S6 & S7 & S9 & S12 & Eth. \\
\hline Water (\%) & 100 & 76 & 60 & 51 & 50 & 49 & 44 & 38 & 35 & 0 \\
\hline Glycérol (\%) & 0 & 24 & 40 & 49 & 50 & 51 & 56 & 62 & 65 & 0 \\
\hline$\mu(\mathrm{mPa} . \mathrm{s})$ & 1 & 2.1 & 3.3 & 4.4 & 5.2 & 6.2 & 7.4 & 9.7 & 12 & 1.2 \\
\hline$\gamma\left(\mathrm{mN} . \mathrm{m}^{-1}\right)$ & 72 & 67 & 65 & 65 & 65 & 64 & 64 & 64 & 64 & 23.5 \\
\hline$\rho\left(\mathrm{kg} . \mathrm{m}^{3}\right)$ & 1000 & 1058 & 1100 & 1120 & 1125 & 1137 & 1141 & 1157 & 1164 & 780 \\
\hline
\end{tabular}

Table 2. Characteristics of the solutions used in the following. Wat stands for Water and Eth for ethanol.

\subsubsection{Experiments}

Our experiment has been designed to be a model experiment of effervescence. It consists in releasing a single air bubble from a needle submerged in a liquid and recording the bubble bursting at the free surface and the ejected droplets. Bubbles are quasi-steadily formed using a syringe pump and detachment frequency is weak enough 
to avoid successive bubbles interaction. Different needle diameters $(5<\Phi(\mu \mathrm{m})<$ $1800)$ allow us to create bubbles with various radii $\left(R_{b}\right)$ ranging from $300 \mu \mathrm{m}$ to 3 $\mathrm{mm}$. This includes most of the range of bubble radii at the surface of a champagne glass, namely $200 \mu \mathrm{m}$ to $1.5 \mathrm{~mm}^{1}$. The bubble collapse, drop velocity and drop size are analyzed through extreme close-up and ultra-fast imagery (Photron SA-5). Depending on the experiment, frame rate is varied from 1000 to 400000 images per second. Macro lenses and extension rings allow us to record with a definition reaching $7 \mu \mathrm{m}$ per pixel. The jet speed $V_{\text {tip }}$ is measured when the tip of the jet reaches the mean water level and the top drop speed $V_{\mathrm{d}}$ is measured when the first drop detaches from the jet. The top drop height is measured using a second digital high-speed camera (Phantom v7.11), triggered by the first camera when the bubble collapses. Its field of view is large enabling to follow the drop all along its trajectory with 1000 frames per second.

\subsection{Dimensionless numbers}

With the aim of establishing the role played by the relevant parameters in the generation of bubble bursting aerosol, we will use three main dimensionless numbers all along this study :

- The Weber number

$$
\mathrm{We}=\frac{\rho V^{2} R}{\gamma},
$$

which compares the effect of inertia and capillarity.

- The Bond number

$$
\mathrm{Bo}=\frac{\rho g R^{2}}{\gamma}
$$

which compares the effect of gravity and capillarity.

- The Morton number

$$
\mathrm{Mo}=\frac{g \mu^{4}}{\rho \gamma^{3}},
$$

which only depends on the fluid properties.

$V$ will be either the jet velocity $V_{\text {tip }}$ or the drop velocity $V_{\mathrm{d}}, R$ will be either the bubble radius $R_{\mathrm{b}}$ or the drop radius $R_{\mathrm{d}}, g$ is the acceleration due to gravity and $\mu$, $\gamma, \rho$ are respectively the liquid viscosity, surface tension and density.

\section{Static bubble shape et film retraction}

\subsection{Shape of static floating bubbles before collapse}

After nucleation, a bubble rises in the liquid because of Archimedes' force until it hits the liquid-air interface. After a few oscillations, the bubble adopts its equilibrium shape at the free surface. This shape results from a balance between two opposing effects: the buoyancy, whose pressure is order of $\rho g R_{b}$, which tends to make the bubble emerge from the free surface and the capillarity, whose pressure is order of $\gamma / R_{b}$, which tends to maintain the bubble below the surface. Comparing these two opposing effect comes down to compute the bubble Bond number, Bo $=\rho g R_{\mathrm{b}}^{2} / \gamma$, which is equivalent to compare the bubble radius with the capillary length of the liquid medium, $l_{g c}=\sqrt{\gamma / \rho g} \simeq 2 \mathrm{~mm}$. Champagne bubbles' radii being always smaller than the capillary length $\left(R_{b}<1.5 \mathrm{~mm}\right)$, the Bond number is lower than 1 and gravity is dominated by capillary effects. Consequently, like a tiny iceberg, a floating bubble 

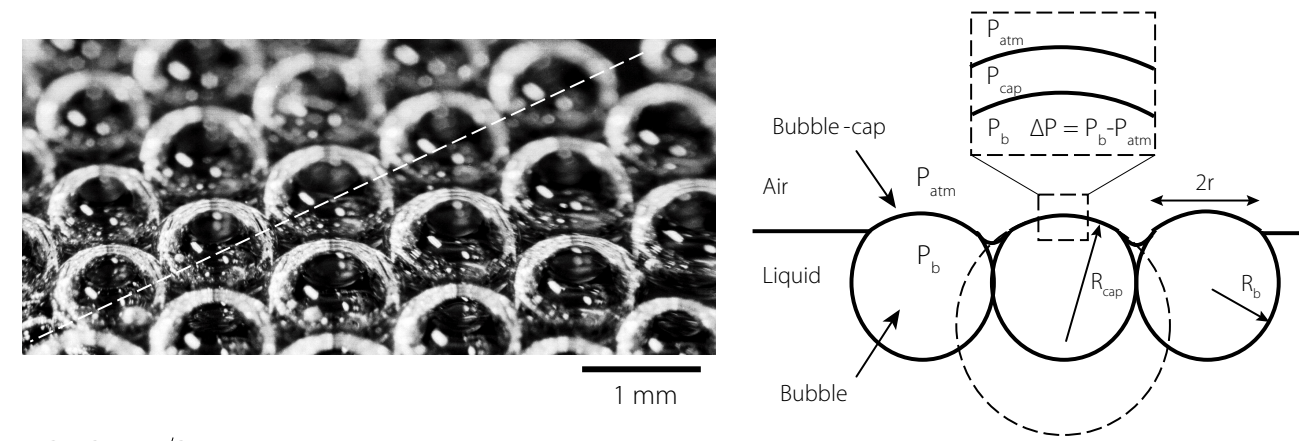

Fig. 5. The snapshot is a view of the bubble monolayer composed of quite millimetric bubbles organized in a hexagonal pattern, each bubble being surrounded by an arrangement of six neighbors; The schematic represent a cross section of three aligned bubbles in touch in the monolayer. $R_{b}$ is the bubble radius, $R_{\text {cap }}$ is the bubble cap radius of curvature and $r$ is the radius of the emerged part of the bubble.

only slightly emerges from the liquid surface, with most of its volume remaining below the free surface.

In the following, the collective effect of bubbles at the surface of a glass of champagne will be not be considered. Typically, we will not take into account interactions between adjoining bubbles (no dimple effects, for example). Let us denote, $R_{\text {cap }}$ and $R_{\mathrm{b}}$, respectively the radii of curvature of the emerged bubble-cap and the submerged bubble. The Laplace law indicates that there is a pressure jump at the interface between the atmosphere and inside the bubble cap : $P_{\text {cap }}-P_{\text {atm }}=2 \gamma / R_{\text {cap }}$, and another one between the cap and the bubble $: P_{\mathrm{b}}-P_{\text {cap }}=2 \gamma / R_{\text {cap }}$ (see schema of Fig. 5 ). This leads finally to a pressure jump between the bubble and the atmosphere

$$
\Delta P=P_{\mathrm{b}}-P_{\mathrm{atm}}=\frac{4 \gamma}{R_{\mathrm{cap}}} .
$$

Moreover, the pressure jump between the submerged part of the bubble and the liquid bulk is $\Delta P=\frac{2 \gamma}{R_{\mathrm{b}}}$. Equalling the 2 pressure jumps yields the following relation between the 2 radius :

$$
R_{\text {cap }}=2 R_{\mathrm{b}} .
$$

The radius of curvature of the emerged bubble-cap is therefore approximately twice that of the submerged bubble volume.

Obviously, the transition between these two zones of different curvature does not appear so abruptly. In order to have a realistic description of the shape taken by a bubble trapped at the air/liquid interface before it bursts, we need to integrate the Laplace equation for each interface coupled with the hydrostatic in the liquid bulk and in the meniscus. Fig. 6 presents the desired profiles obtained by numerical integration of the Laplace equation using MATHEMATICA software. The equation and the numerical method are described in various works ${ }^{4 ; 30 ; 31 ; 32}$. The bubble size are normalized in order to only compare the shape. We observe that the bubble shape changes drastically on this range of size. In particular, the smaller the bubble the more it is spherical and submerged. Note that the radius of a bubble at the surface of a glass of champagne does not exceed $R_{b}=1.5 \mathrm{~mm}$.

Fig. 7 (a) displays an experimental snapshot of a bubble before bursting and the corresponding numerical profile is superimposed with green line. The agreement is perfect meaning that our bubble is at rest before bursting. On the Fig. 6 it is clear that the bubbles are not spherical, so we need to defined an equivalent radius. The 


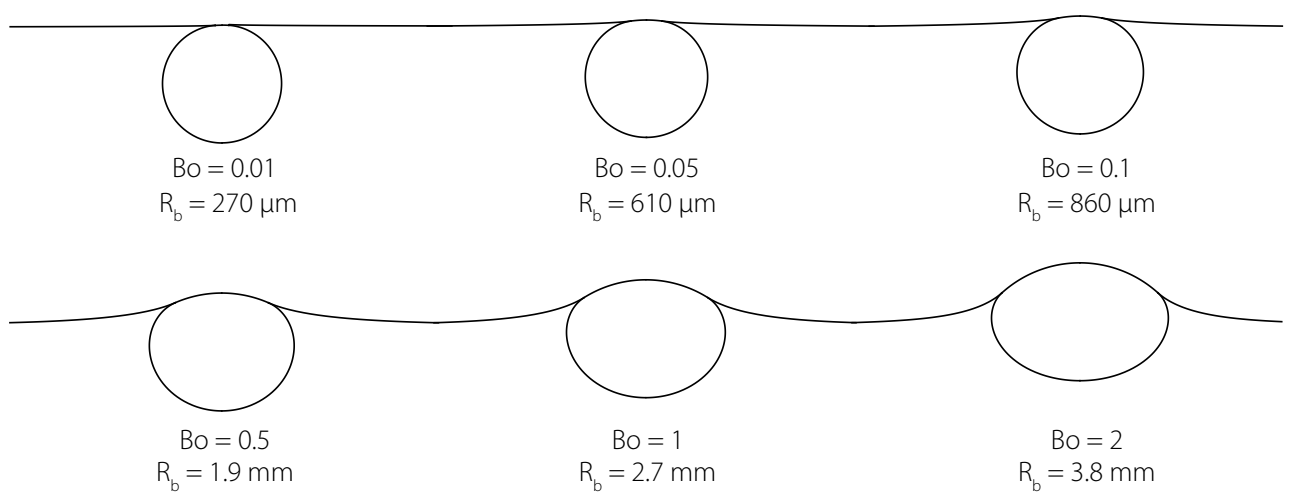

Fig. 6. Profiles of a static floating bubble at the free surface obtained by numerical integration of the Laplace equation using MATHEMATICA software ${ }^{4 ; 30 ; 31 ; 32}$. The smaller the bubble the more it is spherical and submerged. The equivalent size $R_{b}=\left(a^{2} b\right)^{1 / 3}$, defined on the Fig. 7, are indicated below each profile.

red dashed line on Fig. 7 (a) is a semi-elliptic fit of the bottom part of the static bubble. It captures properly the inferior half of the bubble and consequently allows us to define the bubble parameters: $a, b$ and the corresponding bubble radius

$$
R_{b}=\left(a^{2} b\right)^{1 / 3} .
$$

$a$ and $b$ identify respectively the semi-minor and semi-major axis of the ellipsoid.

(a)

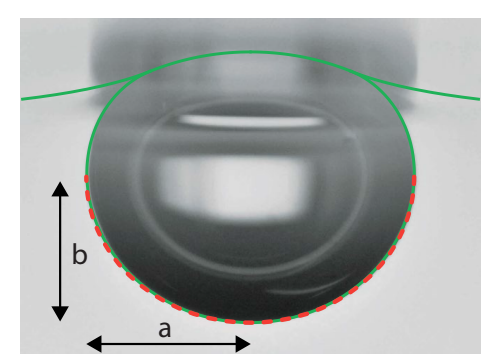

(b)

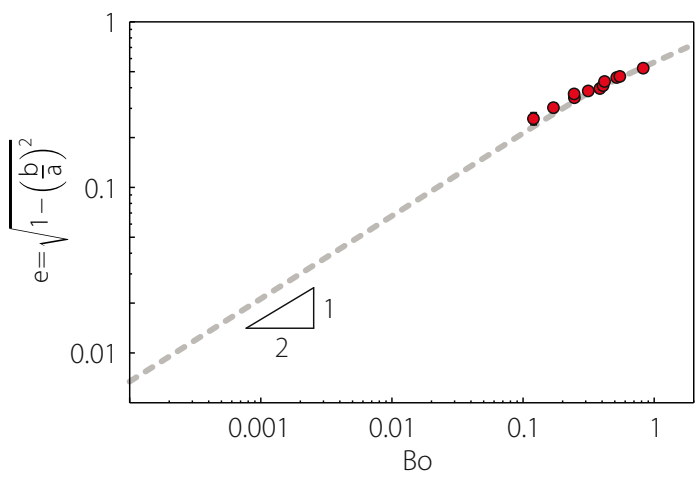

Fig. 7. (a) Picture of a static floating bubble at the free surface. Green profile is obtained by numerical integration of the Young-Laplace equation using MATHEMATICA software ${ }^{4 ; 32}$. The red dashed line is a semi-elliptic fit of the bottom part of the static bubble. $a$ and $b$ identify respectively the semi-minor and semi-major axis of the ellipsoid. (b) Static bubble eccentricity $e=\sqrt{1-(a / b)^{2}}$ computed numerically as a function of the Bond number (dashed line) showing asymptotic behaviors $e \propto \mathrm{Bo}^{1 / 2}$ for small Bond number. The red circles correspond to our experiments in water.

With the aim of quantifying the difference to sphericity, the eccentricity of the static floating bubble, $e=\sqrt{1-(a / b)^{2}}$, computed numerically is plotted versus the 
Bond number with dashed line in Fig.7 (b). The red circles correspond to the eccentricity measured experimentally on bubbles in water. $e$ is found to decrease with the Bond number following $e \propto \mathrm{Bo}^{1 / 2}$, demonstrating the non-sphericity of even small bubbles. This result naturally suggests that, even for Bond numbers lower than 1 , gravity influences the initial bubble shape. We will see later that this might account for the influence of gravity on the subsequent the jet dynamics.

\subsection{Dynamics of a bubble-cap's aperture}

At the free surface, since bubbles' radii are significantly smaller than the capillary length, the liquid films of bubble-caps progressively get thinner due to capillary drainage (capillary pressure dominates hydrostatic pressure). When the liquid film of a bubble-cap reaches a critical thickness, a hole nucleates and the film ruptures, see Lhuissier and Villermaux $(2012)^{4}$ for a complete description of the phenomena.

When the hole appears in the film it is surrounded by a rim which collects the liquid and that propagates very quickly driven by surface tension forces. Balancing inertia with surface tension, Culick proposed the following velocity for the growing hole in a thin rupturing liquid sheet ${ }^{33}$,

$$
V_{\text {Culick }}=\sqrt{\frac{2 \gamma}{\rho e}},
$$

where $e$ is the thickness of the liquid film. The latter expression has already been experimentally confirmed numerous times for the bursting of low viscous thin liquid films ${ }^{4 ; 34 ; 35}$

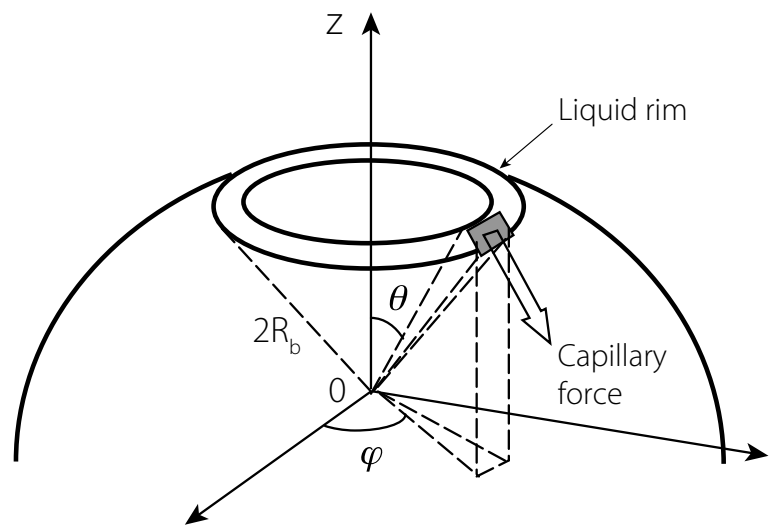

Fig. 8. Schematic representation, in a rupturing spherical bubble-cap with a radius of curvature $2 R_{b}$, of the growing hole surrounded by a rim which collects the liquid and propagates driven by surface tension forces.

In case of a spherical cap with a radius of curvature $R_{\text {cap }}=2 R_{b}$ (see Eq. 5), rupturing from its axis of symmetry (the z-axis) as schematized in Fig. 8, the velocity $u$ of the propagating liquid rim (of mass $M$ ) is ruled by the following equation:

$$
\frac{d(M u)}{d t}=2 \gamma\left(2 R_{b}\right) \sin \theta d \varphi
$$


By replacing in the latter equation, the mass of the liquid rim by its expression, $M=\rho e\left(2 R_{b}\right)^{2}(1-\cos \theta) d \varphi$, the velocity $u$ by $2 R_{b}(d \theta / d t)$ and by developing, one obtains the following differential equation :

$$
\rho e\left(2 R_{b}\right)^{2}\left(\frac{d^{2} \theta}{d t^{2}}(1-\cos \theta)+\sin \theta\left(\frac{d \theta}{d t}\right)^{2}\right)=2 \gamma \sin \theta
$$

Finally, by considering only the constant velocity solution $d^{2} \theta / d t^{2}=0$, one obtains the following expression $u$ for the growing hole,

$$
u=2 R_{b} \frac{d \theta}{d t}=\sqrt{\frac{2 \gamma}{\rho e}}=V_{\text {Culick }}
$$

which is the same as that derived by Culick. The thickness $e$ of a millimetric champagne bubble-cap was already experimentally determined by the classical microinterferometric technique and found to be of order of $10^{-6}$ to $10^{-7} \mathrm{~m}^{36}$. As a result, by replacing in equation (10) the values of $\gamma, \rho$ and $e, u$ is expected to be of order of $10 \mathrm{~m} \cdot \mathrm{s}^{-1}$ for a rupturing champagne liquid film.

The radius of the emerged bubble-cap, $r$, as shown in Fig. 5 , can be estimated by equaling the bubble buoyancy with the capillary pressure inside the thin film as:

$$
\rho g\left(\frac{4}{3} \pi R_{b}^{3}\right) \sim \frac{\gamma}{R_{b}} \pi r^{2} \Rightarrow r \sim \sqrt{\frac{4}{3}} \frac{R_{b}^{2}}{\sqrt{\gamma / \rho g}} \simeq 0.14 \mathrm{~mm} .
$$

With $R_{b}$ taken equal to $0.5 \mathrm{~mm}$. Finally, the characteristic time scale $\tau$ of a millimetric bubble-cap's disintegration should be around:

$$
\tau \sim \frac{r}{u} \sim \frac{1.4 \times 10^{-4}}{10} \sim 10 \mu s
$$

\section{Cavity collapse and jet dynamics}

At this point, we have presented the static bubble shape and the dynamics of bubblecap film retraction. The present section is now dedicated to the collapse of the unstable open cavity and the subsequent jet dynamics. In order to have an overview of the different jet dynamics regimes we will work here with control parameters range broader than the parameters range in champagne. At the end of this section the application in oceanography and in champagne context will be discussed.

The last sixty years have witnessed a number of laboratory studies documenting jet drops properties, such as the ejection speed, the maximum height or the size distribution as a function of bubble volume $\mathrm{e}^{8 ; 37 ; 38 ; 39 ; 40}$. Here we bring a new comprehensive picture of the mechanisms at play in bubble bursting. In particular, the sequence of violent events preluding jet formation ${ }^{5 ; 13}$ and the roles of liquid properties are discussed. This section is extracted from Ghabache et al. $(2014)^{7}$.

\subsection{Jet velocity dependance with bubble size in water}

Figure 9 illustrates a typical jetting event following a bubble bursting at a free surface in water. The top sequence shows the free surface view while the bottom one displays the underwater dynamics. The top sequence takes place between the two first images of the bottom one as indicated by the arrows. The first image of the top sequence shows our static bubble from the top. Then the film separating the bubble from 
the atmosphere drains and bursts leaving the unstable open cavity. This cavity is millimeter-sized so the restoring force which tends to bring this hole back to a flat equilibrium is capillary driven. Bottom sequence displays capillary waves propagating along this cavity and focusing at the bottom. These collapsing waves give rise to the high speed vertical jet shooting out above the free surface as observed on the top sequence. The jet then fragments into droplets due to end pinching mechanism (described in section 5) generating an aerosol of one to ten $\operatorname{droplets}^{8}$.

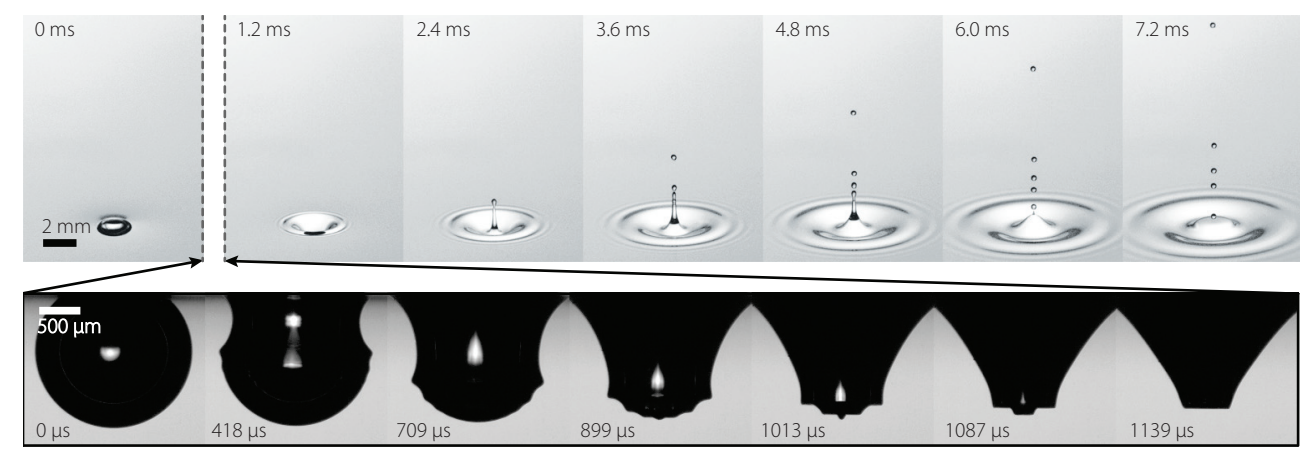

Fig. 9. Time sequence of a typical jetting event following a bubble bursting at a free surface in water. The top sequence shows the bubble bursting event above the free surface, while the bottom sequence displays the bubble under the free surface during the collapse giving birth to the vertical upward jet. The bottom sequence takes place between the two first images of the top one. The times are shown on the snapshots with the same origin.

In order to establish the role played by the relevant parameters in the jet dynamics, we identify the five variables ruling the value of the jet tip velocity:

$$
V_{\mathrm{tip}}=\Phi\left(R_{b}, \rho, \gamma, g, \mu\right)
$$

Using dimensional arguments, this equation becomes a relation between three dimensionless numbers fully describing the jet dynamics:

$$
\mathrm{We}=F(\mathrm{Bo}, \mathrm{Mo})
$$

where, as introduced section 2.3, the Weber number We $=\rho V_{\text {tip }}^{2} R_{b} / \gamma$ compares the effect of inertia and capillarity on the jet dynamics, the Bond number Bo $=\rho g R_{b}^{2} / \gamma$ compares the effect of gravity and capillarity on the initial bubble and the Morton number Mo $=g \mu^{4} / \rho \gamma^{3}$ only depends on the fluid properties and is in particular independent of the bubble radius $R_{b}$. Various scaling relations for the velocity are reported in the literature, ranging from exponential fits of experimental data ${ }^{40}$ (see curved dashed line Fig. 10) to algebraic laws $V_{\text {tip }} \propto R_{b}^{-1 / 2}$ in numerical simulations disregarding gravity ${ }^{5}$. This diversity certainly emphasizes the need for further experimental analysis.

We set out by investigating experimentally in Fig. 10 the dependence of $V_{\text {tip }}$ with $R_{b}$ in a log-log plot. Our experimental data (circles) rest along the line $V_{\text {tip }} \propto R_{b}^{-1}$, as indicated by the red dashed line fitting the experimental velocities. Note that bubbles with $\mathrm{Bo}>1$ (radii greater than $3 \mathrm{~mm}$ ) are out of scope of this study because they give rise to jets with a different dynamics and would constitute an other study. On the same figure various data from the literature have been plotted: the top drop velocity measured experimentally in fresh water ${ }^{40}$ or in sea water ${ }^{8}$, and the maximum tip velocity of the jet computed numerically in fresh water ${ }^{39}$. Regardless of some slight 
differences they all follow the same trend $V_{\text {tip }} \propto R_{b}^{-1}$. This specifies the form of the Eq.(14) providing the variation with Bond number, yielding:

$$
\mathrm{We}=\mathrm{Bo}^{-1 / 2} f(\mathrm{Mo}) \text {. }
$$

The $R_{b}^{-1}$ behavior is the footprint of gravity effects. The introduction of a second length scale, the gravity-capillary length $\ell_{\mathrm{gc}}=\sqrt{\gamma / \rho g}$, allows departures from capillaro-inertial predictions through length scales ratios ${ }^{41}$ : We $=\left(\ell_{\mathrm{gc}} / R\right) f(\mathrm{Mo})$. Though the Bond number remains small in the experiments, the gravity plays a genuine role in the collapse dynamics through the initial static bubble shape as described in paragraph 3.1. Note that Froude number of the jet at the mean water level is $\mathrm{Fr}=V_{\mathrm{tip}} / \sqrt{g R_{b}}=\sqrt{\mathrm{We} / \mathrm{Bo}}$, and can be expressed here as $\mathrm{Fr}=\mathrm{Bo}^{-3 / 4} f(\mathrm{Mo})^{1 / 2}$. In water, with the Bond values of Fig.10 one obtains Fr $\in[7-160]>1$, indicating that gravity hardly affects the jet dynamics at least before eruption confirming the role of the initial shape.

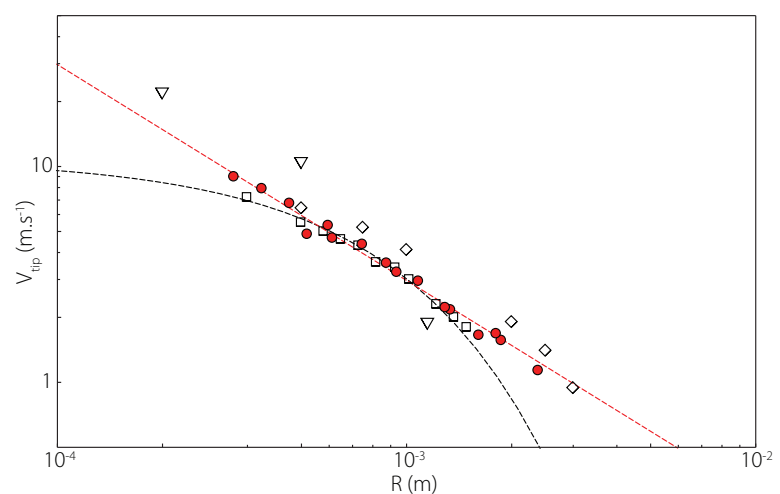

Fig. 10. Jet velocity $V_{\text {tip }}$, measured when the jet passes the free surface level, as a function of the bubble radius $R$ in water from our experiments (red circle) and data from the literature: (square) top drop velocity ${ }^{40}$ along with the exponential fit represented by the curved dashed line $V=10.72 \mathrm{e}^{-1.27 .10^{3} R}$ measured experimentally in fresh water, (diamonds) maximum tip velocity computed numerically in fresh water ${ }^{39}$ (triangle) top drop velocity ${ }^{8}$. The red dashed line is a fit of our experimental data exhibiting the common trend $V_{\text {tip }}=\xi R^{-1}\left(\xi=2.9510^{-3}\right.$ $\left.\mathrm{m}^{2} \cdot \mathrm{s}^{-1}\right)$. The curve dashed line is the exponential fit $V=10.72 \mathrm{e}^{-1.27 \cdot 10^{3} R}$ given by Spiel $e t$ $a l .{ }^{40}$.

\subsection{Drop velocity versus jet velocity}

We chose here to work with the the jet velocity as we considered that it was better defined than the drop velocity (same altitude, no drop detachment process). However, it is the drop velocity which will be required for the quantification of aerosol evaporation. In Fig. 10 it is remarkable that our jet velocities match the top drop velocities found in the literature (square ${ }^{40}$ and triangle ${ }^{8}$ ). This is important as it makes our results presented here quantitatively relevant for drop dynamics on a large range in bubble radius. To go further in the comparison between drop and jet velocity we present in Fig. 11 the jet and the drop velocities resp. $\mathrm{V}_{\text {tip }}$ and $\mathrm{V}_{\mathrm{d}}$, as a function of the bubble radius $R_{b}$ in water. We recall that $V_{\text {tip }}$ are $V_{d}$ are respectively measured when jet passes the free surface and when the drop detaches. We find again that the 
two velocities are very close to each other for bubble radii up to $\sim 1.2 \mathrm{~mm}$. However, for greater radii the drop velocity becomes lower than the jet velocity leaving the $\mathrm{R}_{b}^{-1}$ regime as showed on the log-log plot in the top right inset. Anyway, as most of the bubbles at the surface of a champagne glass are smaller than $1.2 \mathrm{~mm}$ radius they produce drops with the same velocity as their jet.

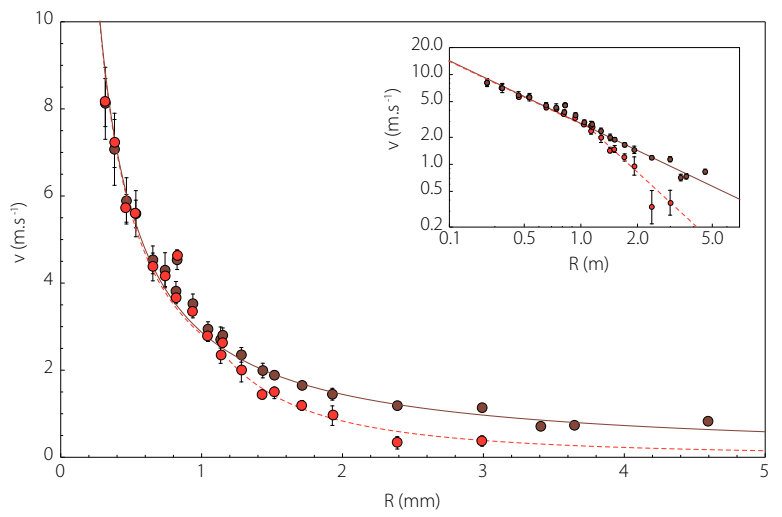

Fig. 11. Jet and first drop velocity, resp. $V_{\text {tip }}$ and $V_{d}$, as a function of the bursting bubble radius $\mathrm{R}_{b}$ in water from our experiments. The line curve fits the experimental values of the jet velocity while the dashed line curve is a fit of the first drop velocity. On the top right inset the same graph is represented in a log-log plot. The line exhibits the common trend for the jet velocity $\mathrm{V}_{\text {tip }} \propto \mathrm{R}_{b}^{-1}$ and the dashed line curve quits this trend for bubble radius greater than $\sim 1.2 \mathrm{~mm}$.

\subsection{Jet velocity dependance with liquid properties}

We now investigate how the jet eruption velocity $V_{\text {tip }}$ depends on the liquid properties and therefore on the Morton number. The Weber number is plotted as a function of the Bond number for various Morton number on Fig. 12 (a). To browse the Morton range we mainly change the liquid viscosity (see table of Fig. 12). The first clear observation is that the jet dynamics depends on the viscosity although the jet Reynolds number is greater than 1. Furthermore, the regime $\mathrm{We} \propto \mathrm{Bo}^{-1 / 2}$ is retained on around four decades in Morton number, from $1 \mathrm{mPa} . \mathrm{s}$ to around $7.5 \mathrm{mPa} . \mathrm{s}$, all plotted with filled markers. This defines the boundary of our study considering that this viscous regime characterized by $\mu \gtrsim 9 \mathrm{mPa}$.s and showed with empty markers is out of the scope of this paper. Finally, for values of viscosity less than $6 \mathrm{mPa}$.s we observe a surprising increase of the Weber number with Morton number, meaning that for a given bubble radius in this range, the jet is drastically faster as the liquid viscosity is increased.

The non-dimensional jet velocity $\mathrm{We} \mathrm{Bo}^{1 / 2}$ is plotted as a function of the Morton number on Fig. 12(b), therefore specifying $f(\mathrm{Mo})$ (see Eq. (15)). A bell shaped curve is clearly observed with a maximum for $\mu=5.2 \mathrm{mPa}$.s. To illustrate this unexpected behavior we display inside Fig. 12 four snapshots of the jet at the same dimensionless time $\left(\mathrm{t} / \sqrt{\rho R^{3} / \gamma}=1 / 5\right)$, same Bond number $(\mathrm{Bo} \simeq 0.14)$ but four different Morton numbers. The jet morphology undergoes a neat qualitative change as the liquid gets more viscous: the jet first becomes thinner, detaching more and smaller droplets and then ends up fat and small for high Morton number. 

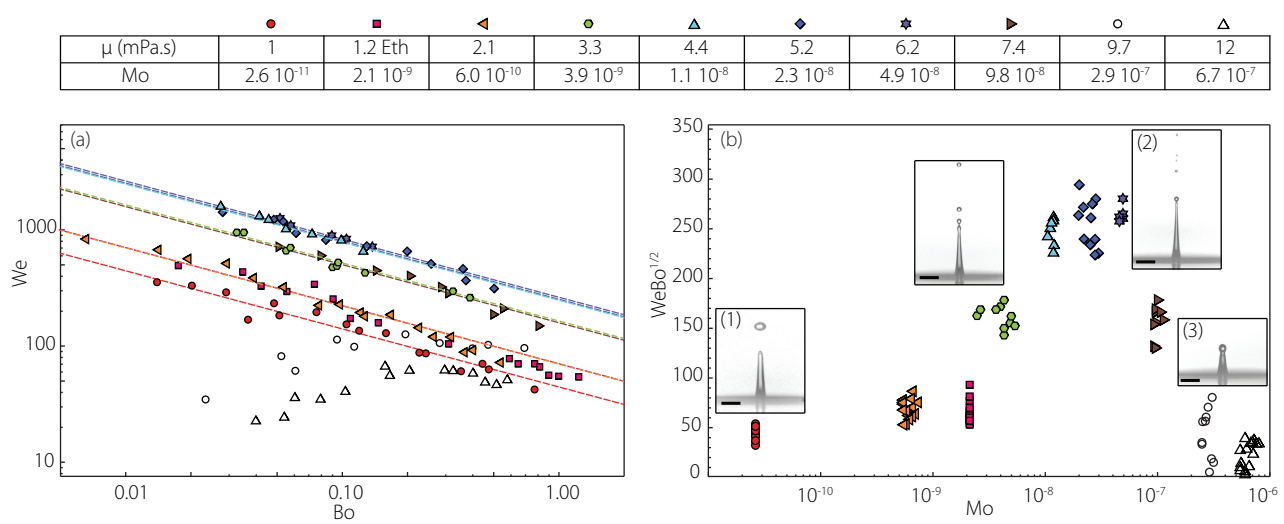

Fig. 12. Table: Value of the liquid viscosity and of the associated Morton number corresponding to each symbol. Eth. stands for Ethanol . (a) Weber number as a function of the Bond number for various values of the Morton number. All the colored symbols follow the same trend $\mathrm{We} \propto \mathrm{Bo}^{-1 / 2}$ as showed by the dashed lines. (b) $\mathrm{We} \mathrm{Bo}^{1 / 2}$ as a function of the Morton number Mo. Four snapshots display the typical jet observed at the same dimensionless time $\left(\mathrm{t} / \sqrt{\rho R^{3} / \gamma}=1 / 5\right)$, same Bond number $(\mathrm{Bo} \simeq 0.14$ ) and four different Morton numbers corresponding to red circle, green hexagon, purple star, empty triangle. The black bar represents $500 \mu \mathrm{m}$.

\subsection{Capillary wave dynamics and cavity collapse}

In order to grasp the mechanisms leading to such a particular dynamics, we now turn to the jet formation by focusing on the cavity collapse per se. Lower sequence of Fig. 9 displays a typical bubble collapse in water, where a train of capillary waves propagates, converges to the nadir (bottom of the cavity), and gives rise to the jet. Fig. 13 shows a temporal zoom of the last microseconds before the cavity collapses $\left(t \simeq t_{0}\right)$ for three different Morton numbers and same Bond number. These three sequences (a), (b) and (c) are the cavity collapse leading to the three jets (1), (2) and (3) displayed on Fig. 12. On the last image of each sequence the cavity is reversed and the upward jet (not seen on the picture) is developing. These sequences show that the cavity reversals are very similar between the 6.2 and $12 \mathrm{mPa}$.s solutions and drastically different from water. In particular the small capillary waves present in the water collapse (a) have disappeared for higher viscosities (b) and (c). It has been shown in numerical simulation ${ }^{5}$ and in other experimental contexts ${ }^{42 ; 43}$ that such collapse exhibits a self-similar dynamics that can lead in some cases to very thin and rapid jets. In such a situation, the cavity collapses through a nonlinear balance between capillary force and inertia, leading to a self-similar behavior where the lengths scale like $\left(\gamma\left(t_{0}-t\right)^{2} / \rho\right)^{1 / 3}\left(t_{0} \text { corresponding to the instant of the singular collapse }\right)^{44}$. In the three cases presented here, the same self-similar collapse is clearly at play, as shown on Figs 13(d,e) where the different cavity profiles plotted at different times before $t_{0}$ collapse when lengths are divided by $\left(t_{0}-t\right)^{2 / 3}$. So we observe a capillaryinertia self-similar collapse for each case, even though the jets show clear differences (see Fig. 12 (1), (2) and (3)) and are not singular. Interestingly, shapes, timescales and overall self-similar evolution for the collapsing cavity are identical for the high viscosity cases (b) and (c) as shown in Fig. 13 (e). The fact that jet velocity falls with increasing viscosity therefore does not depend on the details of the cavity collapse, but more likely on the large viscous stresses developing in the highly strained region at the jet root. However, these collapses are strongly different from the collapse in water 
(a) which is perturbed by the presence of the small capillary waves. These waves are always traveling on top of the interface and are inherent to the complex dynamics. But we observe that increasing the viscosity leads to smoothing the collapse. In particular the closest time to $t_{0}$ in water, represented by the dashed profile, does not coalesce properly, signifying that the dynamics is no more self-similar. This results in a collapse leaving its self-similar regime sooner than in a more viscous case, when the remnant ripples are damped. By defining $\mathrm{L}_{\text {min }}$ as the width of the small left cavity when the collapse just quitted it self-similar behavior, Fig. 13 (f) shows the variation of this effective collapsing cavity giving rise to the jet and reveals that it decreases with Morton number. This agrees with the idea of a self-similar collapse getting closer to the singularity as viscosity is increased and justifies why the jet velocity is increasing with Morton number.

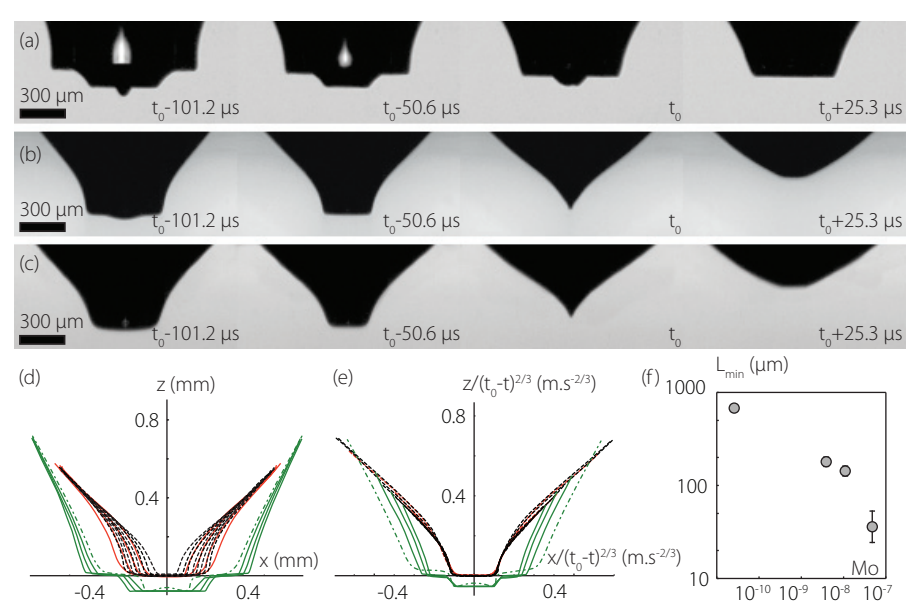

Fig. 13. Snapshots of the final stage of the cavity collapse before the jet develops. (a), (b) and (c) correspond respectively to the jets (1), (2) and (3) of Fig. 12. $t_{0}$ identifies the time of the wave collapse giving rise to the jet. The cavity profiles corresponding to the three sequences are plotted at different times on (d) (green for sequence (a), black for (b) and red for (c)). (e) shows the collapse of these profiles according to the capillary-inertia self-similar behavior where lengths scale with $\left(t_{0}-t\right)^{2 / 3}$. (f) $\mathrm{L}_{\text {min }}$ versus Morton number. $\mathrm{L}_{\text {min }}$ defines the width of the small left cavity when the collapse just quitted it self-similar behavior. The size of this effective cavity giving rise to the jet eruption decreases with viscosity for $\mu \lesssim 6$ mPa.s.

This suggests an original mechanism to explain the role of the capillary waves for small Morton numbers. We consider that these waves break the self-similar dynamics when they are large enough (typically, when the wave amplitude is of the order of the self-similar structure). Because the phase velocity of the capillary waves yields $c \propto \sqrt{\gamma k / \rho}$, the shorter the wave, the faster it converges to the nadir, suggesting that the singular dynamics is destroyed by small waves first. This picture has now to be corrected by the viscous damping of the capillary waves ${ }^{45}$ which is also increasing with the wave number (with damping rate $\propto \mu k^{2} / \rho$ ). Therefore, as the viscosity increases, the interface is smoothed near the nadir and the instant where the oscillations destroy the self-similar dynamics is delayed closer and closer to the singularity.

Finally, two regimes of the jet dynamics as a function of the Morton number have been pointed out: for Mo $\lesssim 3 \cdot 10^{-8}$ the viscosity promotes the jet velocity by smoothing the collapsing cavity and for $\mathrm{Mo} \gtrsim 3 \cdot 10^{-8}$ the jet velocity decreases with 
viscosity. At the frontier of these two regimes the jet is very thin and its velocity is maximal.

\subsection{Conclusion and applications : from oceanography to champagne}

\section{(a) VISCOSITY : 1 mPa.s}
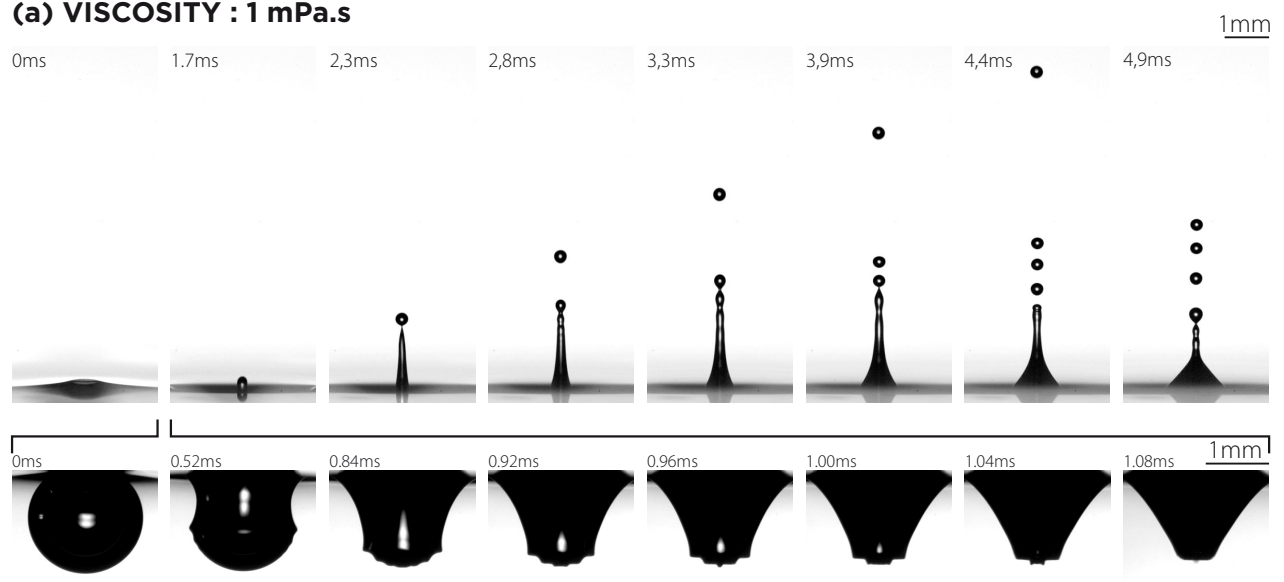

(b) VISCOSITY : $6 \mathrm{mPa} . \mathrm{s}$

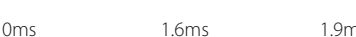

$1.9 \mathrm{~ms} \quad 2.1 \mathrm{~ms}$

$2.4 \mathrm{~ms} \quad 2.7 \mathrm{~ms}$

$2.9 \mathrm{~ms} \quad 3.2 \mathrm{~ms}$

$\underline{1 \mathrm{~mm}}$

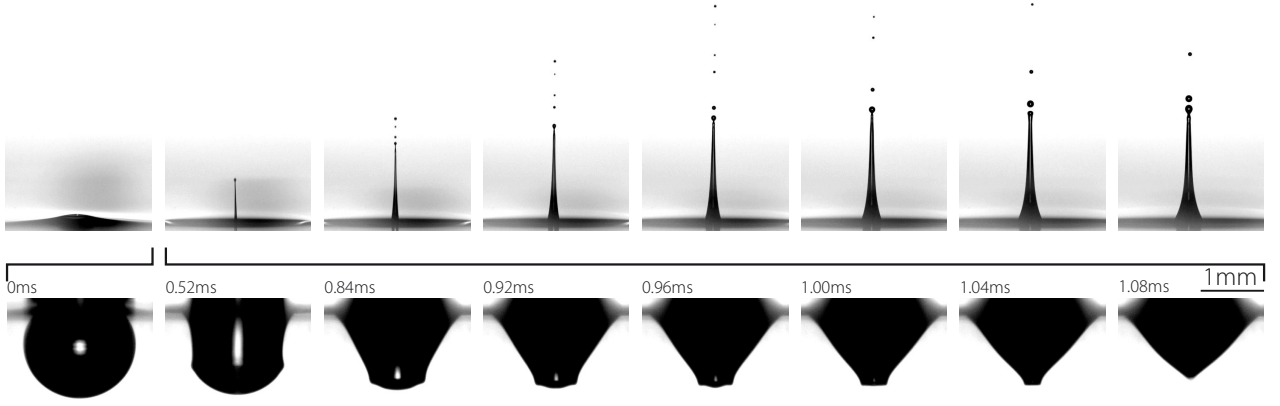

Fig. 14. Experimental time sequences of two typical jetting events following a bubble bursting at a free surface in (a) water (b) glycerol water mixture six times more viscous than water. In each case, the top sequence shows the bubble bursting event above the free surface, while the bottom sequence displays the bubble under the free surface during the collapse giving birth to the vertical upward jet. The bottom sequence takes place between the two first images of the top one. Space scale is on each sequence and times are shown on the snapshots with the same origin. Bubble radius are : (a) $R_{b}=884 \mu \mathrm{m}$ (b) $R_{b}=915 \mu \mathrm{m}$.

As a conclusion and to sum up the influence of the fluid properties on the drop properties, Figure 14 illustrates a typical jetting event following a bubble bursting at a free surface in water and in a six times more viscous solution. In each case, the bubble has the same radius and the top sequence shows the view above the free surface while the bottom one, that takes place between the two first images of the top one, displays the underwater dynamics. We observe that the jets are drastically different in the two 
different solutions. In the viscous solution the jet is much thinner, detaches smaller droplets and is faster than in water. This means that by changing the viscosity, or more generally the Morton number, we can produce very different bubble bursting aerosol. The mechanism leading to such a counter-intuitive dynamics comes from the underwater collapsing cavity. Indeed, we observe that the small capillary waves present in the water collapse have been damped in more viscous liquids. Consequently, the viscous cavity collapse, cleared of capillary ripples, can thus be more intense by getting closer to its singularity, enabling the generation of a faster and thinner jet.

These differences on the aerosol production are quantitatively shown on the bell shaped curve of Fig. 12. This curves presents a maximum for Mo $=2.10^{-8} \mathrm{mPa} . \mathrm{s}$, and on the left of this maximum corresponds the range of liquid properties where the drop velocity increases with the Morton number, Mo $=g \mu^{4} / \rho \gamma^{3}$. This curve has been reproduced in gray on Fig. 15. On top of this gray curve, the red points correspond to bubbles bursting in water and the yellow, the green and the blue ones correspond to the hydro-alcoholic solutions mimicking the champagne at three different temperature from $20^{\circ} \mathrm{C}$ (yellow) to $4^{\circ} \mathrm{C}$ (blue). More generally, the blue zone corresponds to the Morton range existing in oceanography and the yellow zone the range of Champagne wine. The marine aerosols are then in the flat region of the jet velocity dependance on Mo, meaning that hydrodynamical properties of ocean, notably changing with temperature, barely affects the droplet ejection velocity. However, it is then extremely interesting to see that the champagne lies on the zone of the curve where a slight change of liquid properties leads to a drastic change of the drop velocity/size and consequently on the subsequent aerosol characteristics. These results are then crucial in the context of champagne industry. Indeed, they suggest that the characteristics of bursting bubble aerosols in champagne could be tuned through the champagne properties. The question we want to eventually address is, can we find liquid properties that would enable to optimize the aerosol evaporation? With this aim, and in order to improve our understanding of the whole effervescence process, the next step is to study the drops detachment as a function of bubble radius and liquid properties.

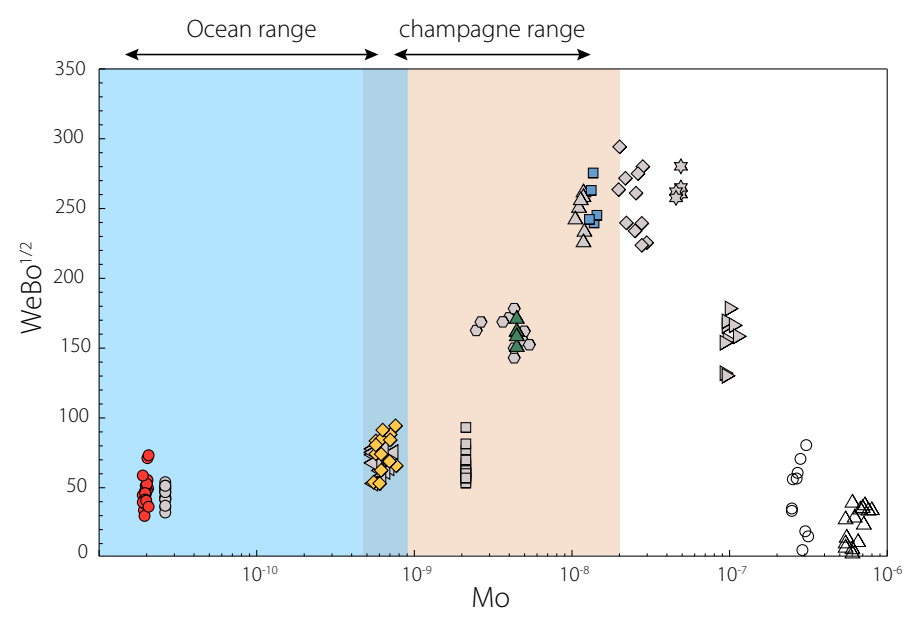

Fig. 15. Non-dimensional jet velocity $\mathrm{We} \mathrm{Bo}^{1 / 2}$ as a function of the Morton number Mo for various values of the morton number, namely for various liquid properties. The gray points correspond to the liquid used in the previous study ${ }^{7}$ while the colored points are those presented in the present work, especially the yellow, the green and the blue ones which corresponds to the hydro-alcoholic solutions mimicking the champagne at three different temperature from $20^{\circ} \mathrm{C}$ (yellow) to $4^{\circ} \mathrm{C}$ (blue). The red points correspond to bubbles bursting in water. 


\section{End pinching and top drop size}

As we saw before, no film drops are produced above a glass of champagne and dynamics of jet drops is strongly modified by liquid parameters. Furthermore, as we will see later, the top jet drops, which bound the edge of the aerosol cloud, dominate the evaporation process as they are faster and usually bigger than the others or with a comparable size. Therefore, in this section, the size of the top jet drop produced by bubble bursting is investigated as a function of the mother bubble size ${ }^{9}$ and the liquid properties (see Fig. 16). Scaling laws for the drop diameter along with their regime of existence will be determined, discussed and the mechanisms will be interpreted. As in the previous section, we will work here with control parameters range broader than the parameters range of Champagne wine in order to gain an complete view of the different regimes. This section is extracted from Ghabache et al. $(2016)^{10}$.

\subsection{The end-pinching mechanism}

An infinite cylinder of liquid at rest, subjected to the influence of surface tension, will break up into a number of individual droplets through the so-called Rayleigh-Plateau instability. The bubble bursting jets, depicted in Fig. 16, are finite and do not break as a consequence of Rayleigh-Plateau instability. Instead, the breakup takes place at the jet tip and detaches one drop at a time. This mechanism, called end-pinching, consists of a competition between the capillary retraction of the jet tip, shaping a blob $^{46}$, and a pressure-driven flow from the cylindrical jet toward the bulbous end. This leads to the development of a neck, where the jet joins the blob, and thus to the drop detachment via a capillary pinch off process. This mechanism has been first described in the context of a strongly deformed viscous drop ${ }^{47}$ and later for a free liquid filament of arbitrary viscosity ${ }^{48}$. This end-pinching capillary breakup of liquid jets is important in several industrial contexts, especially because of the broad range of applications of inkjet printing technology. Indeed, it enables accurate drop deposition of liquids, and includes production of organic thin-film transistors, Liquid Crystal Displays (LCD), fuel or solar cells, Printed Circuit Boards (PCB), dispensing of DNA and protein substances, or even fabrication of living tissue ${ }^{49}$. Recently, the end-pinching of a stretched inertially driven jet shooting up after a cavity collapse has been described theoretically and numerically ${ }^{50}$. These stretched jets are found in many situations ${ }^{41 ; 51}$, in particular bubble bursting, and they all have similar properties.

\subsection{Top jet drop size variation}

Figure 16 presents the jet and released drop shape following bubble bursting. In the cases where no drop detaches the jets are displayed at their maximum height. On the $\mathrm{x}$ and $\mathrm{y}$-axis the jets and drops shape is represented, respectively, for six different liquid viscosities and three different mother bubble radii. It is clear on this diagram that, independently of the viscosity, the bigger the bubble the bigger the top drop. This intuitive result has been observed in water in various previous studies ${ }^{9}$. Although the variation with viscosity has been qualitatively seen in the previous section (see snapshots of Fig. 12 (b)), it is still quite unexpected. Indeed, irrespectively of the bubble radius in the range considered here, the top drop shrinks as viscosity is increased, and seems to reach a minimum for a liquid viscosity around 6-7 mPa.s here. For higher viscosities, no drop is detached, in accordance with previous study ${ }^{52}$. This decrease of the drop radius with viscosity is surprising, in particular because 


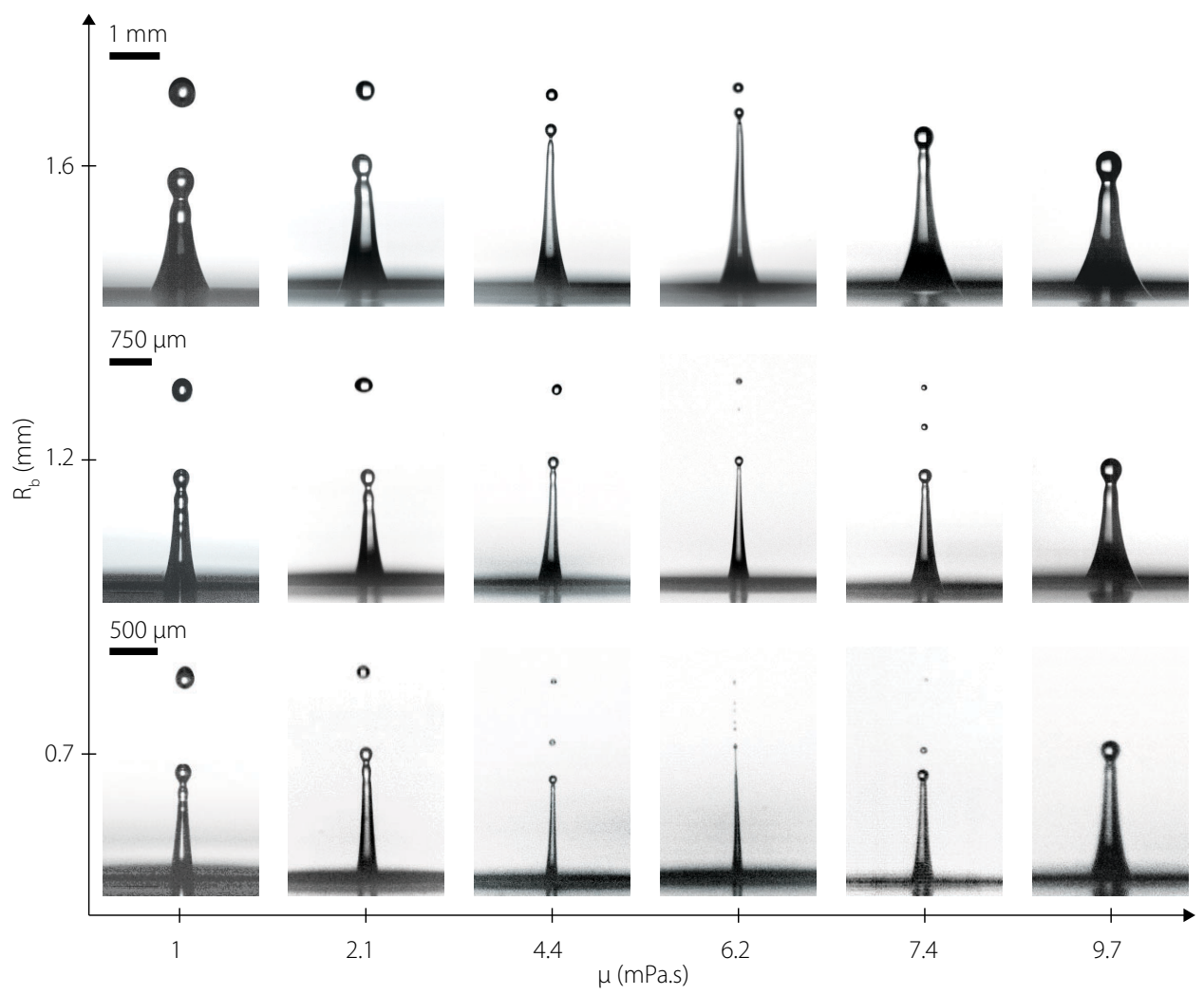

Fig. 16. Snapshot of a typical jetting event following a bubble bursting at a free surface. The jets and drops shape is displayed for three mother bubble radii, reported on the y-axis, bursting in water and five water-glycerol mixtures of viscosity indicated on the x-axis. For those six solutions, the surface tension is almost constant (ranging from 64 to $72 \mathrm{mN} . \mathrm{m}^{-1}$ ) so that one mainly observes in this figure the effect of changing viscosity. top drop size decreases with bubble radius and increasing liquid viscosity. The biggest drop, on the top left corner of this diagram, is about $400 \mu \mathrm{m}$ radius and the smallest $\left(R_{b}=0.7 \mathrm{~mm}\right.$ and $\mu=$ $7.4 \mathrm{mPa} . \mathrm{s})$ reached $20 \mu \mathrm{m}$. The scale bar is showed on the top left corner of each bubble radius and is the same the whole row.

the Ohnesorge number based on the drop radius, namely $\mathrm{Oh}=\mu / \sqrt{\rho R_{d} \gamma}$, which compares the effect of viscosity and capillarity, is included between $10^{-1}$ and $10^{-3}$ and is consequently always lower than 1 . This therefore suggests that viscous effects should be neglected in the description of jet breakup, as done in similar cases ${ }^{50}$. We will see further down why, in this particular case of bubble bursting jet, the liquid viscosity has such a strong influence.

\subsection{Top jet drop radius and bubble bursting}

We now plot, in Fig. 17 (a), the variation of the top drop radius $R_{d}$ as a function of the mother bubble radius $R_{b}$ for different values of the liquid parameters $(\mu, \gamma$ and $\rho$ ) indicated in the table above. This quantifies our previous observation of drop shrinking with decreasing bubble radius and increasing liquid viscosity, from $400 \mu \mathrm{m}$ to $20 \mu \mathrm{m}$ for the solutions plotted here. We also observe that the same drop shrinking occurs when surface tension is decreased. Moreover, it appears that, regardless of 

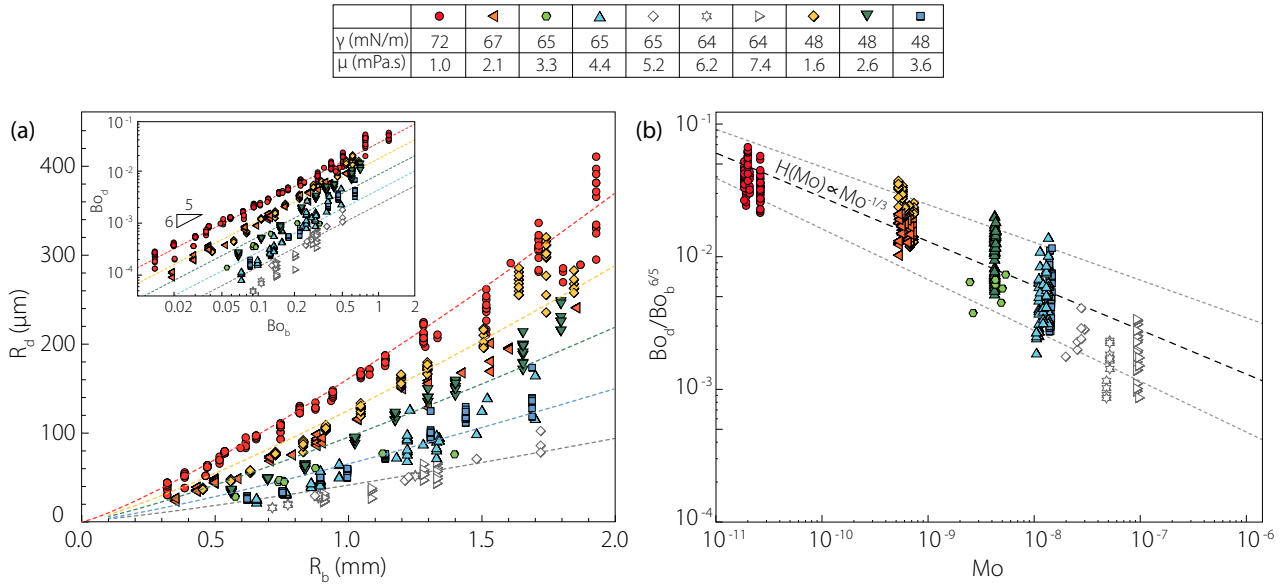

Fig. 17. (a) top drop radius as a function of the mother bubble radius for bubble bursting in liquids with different surface tension and viscosity. The parameters of these liquids associated to the corresponding symboles are summarized in the table above the graph. In the inset, the Bond number built on the drop radius is plotted as a function of the Bond number built on the bubble radius for the same liquids. The dashed lines represent $R_{d} \propto R_{b}^{6 / 5}$ in the graph and $B o_{d} \propto B o_{b}^{6 / 5}$ in the inset. (b) $\mathrm{Bo}_{d} / \mathrm{Bo}_{b}^{6 / 5}$ as a function of the Morton number. The dashed line fits the experimental data plotted with closed symbols, up to Mo $\sim 10^{-8}$, following the trend $\mathrm{Bo}_{d} / \mathrm{Bo}_{b}^{6 / 5} \propto \mathrm{Mo}^{-1 / 3}$. The error bar on the exponent is $1 / 20$, and the two bounds $\mathrm{H}(\mathrm{Mo}) \propto \mathrm{Mo}^{-1 / 3 \pm 1 / 20}$ are plotted on the graph with dotted line.

the liquid parameters considered in this graph, the drop size increases with bubble radius following roughly the same variation for all the curves : $R_{d} \propto R_{b}^{6 / 5}$, shown with dashed lines on the graph. Note that the historical law, proposed for the top jet drop radius produced by bubble bursting in water, that predicts a drop radius being the tenth of the bubble radius $\left(R_{d}=R_{b} / 10\right)^{53}$, is only valid for bubble radii smaller than five hundred micrometers. More accurate laws have been written ever since. In particular, when $R_{b} \geq 0.1 \mathrm{~mm}$, the relationship $R_{d}=0.075 R_{b}^{1.3}$ has been proposed, with radii expressed in millimeters ${ }^{54}$. This variation is very closed to the one we find.

Thanks to our experimental relationships between $R_{d}$ and $R_{b}$, we are now able to write a more universal scaling law, taking into account the liquid parameters. It is clear that top drop size depends on bubble radius $R_{b}$, liquid viscosity $\mu$ and we assume that surface tension $\gamma$, density $\rho$ and gravity $g$ might also influence its selection, yielding :

$$
R_{d}=\Pi\left(R_{b}, \rho, \mu, \gamma, g\right)
$$

Using dimensional arguments, this equation becomes a relation between three dimensionless numbers fully describing the top drop size selection :

$$
\mathrm{Bo}_{d}=F\left(\mathrm{Bo}_{b}, \mathrm{Mo}\right)
$$

where the Bond numbers $\mathrm{Bo}_{d}=\rho g R_{d}^{2} / \gamma$ and $\mathrm{Bo}_{b}=\rho g R_{b}^{2} / \gamma$ compare the effect of gravity and capillarity on the top drop and the initial bubble respectively, and the Morton number $\mathrm{Mo}=g \mu^{4} / \rho \gamma^{3}$ only depends on the fluid properties. On the inset of Fig. 17 (a) the variation of the top drop Bond number is plotted as a function of the mother bubble Bond number for the same solutions as in Fig. 17 (a). The variation $\mathrm{Bo}_{d} \propto \mathrm{Bo}_{b}^{6 / 5}$ is also plotted with dashed lines. This power law, independent of the liquid parameters, still works reasonably well, allowing us to write the following 
scaling law :

$$
\mathrm{Bo}_{d}=\mathrm{Bo}_{b}^{6 / 5} H(\mathrm{Mo}) .
$$

With the aim of estimating the dependance of the drop size with the liquid properties, namely $\mathrm{H}(\mathrm{Mo}), \mathrm{Bo}_{d} / \mathrm{Bo}_{b}^{6 / 5}$ is plotted as a function of the Morton number on Fig. 17 (b). We observe that the data with closed symbols gather along a line, up to $\mathrm{Mo} \simeq 10^{-8}$, corresponding to a viscosity $\mu=5.2 \mathrm{mPa}$.s for a water-glycerol mixture. This line is properly fitted by $\mathrm{H}(\mathrm{Mo})=\mathcal{A} \mathrm{Mo}^{-1 / 3}$ with $\mathcal{A}=1.110^{-5}$. As the results are slightly scattered we estimate the error bar by fitting the lower and upper bounds and we find $\mathrm{H}(\mathrm{Mo}) \propto \mathrm{Mo}^{-1 / 3 \pm 1 / 20}$, these two bounds are plotted on the graph with dotted line. Therefore, in this regime, ranging on around three decades in Morton number, and including the champagne range, we established a scaling law for the top drop size as a function of the bubble radius and liquid parameters, in the context of bubble bursting :

$$
\mathrm{Bo}_{d}=\mathcal{A B o}{ }_{b}^{6 / 5} \mathrm{Mo}^{-1 / 3} .
$$

This result is essential because the bubble radius and the liquid parameters are the natural experimental parameters for bursting bubble aerosol measurement. In particular, the size distribution of bubbles is known in ocean ${ }^{55 ; 56}$ and can even be controlled in a glass of champagne ${ }^{57}$.

However, under this form, Eq. (19) is delicate to interpret, in particular, the confusing role of viscosity, that is expected to be negligible $(\mathrm{Oh} \ll 1)$. In addition, this scaling law contains substantial experimental data scattering due to an accumulation of variability, when the jet is created and when the drop is detached. In the following, we wish therefore, to express the drop radius as a function of only jet parameters, typically by disposing of the bubble radius.

\subsection{Top jet drop radius and jet dynamics}

When a bubble collapses, a jet is formed with a given shape, tip velocity, local strain rate etc. In this regime, where Mo $\lesssim 10^{-8}$, the decrease of the drop size with Morton number comes along with a thinning down of the whole jet and an increase of the jet velocity. This has been largely discussed in section 4 and the corresponding scaling law, for the jet velocity as a function of the bubble radius and liquid parameters, has been proposed : $\mathrm{We}_{b}=\mathrm{Bo}_{b}^{-1 / 2} f(\mathrm{Mo})$ with the Weber number $\mathrm{We}_{b}=\rho V_{\text {tip }}^{2} R_{b} / \gamma$ and $V_{\text {tip }}$ the jet tip velocity as the jet passes the free surface level. Therefore, with the aim of decoupling the effects of bubble collapse and jet dynamics on the drop detachment, $\mathrm{We}_{b}=\mathrm{Bo}_{b}^{-1 / 2} f(\mathrm{Mo})$ is combined with Eq. (19) in order to eliminate the bubble radius. This eventually yields the following scaling law relating the drop radius, the jet velocity and the liquid parameters :

$$
\mathrm{Bo}_{d}=\left(\mathrm{Fr}_{d} \mathrm{We}_{d}\right)^{-3 / 5} G(\mathrm{Mo})
$$

where $\mathrm{Fr}_{d}=V_{\text {tip }}^{2} / g R_{d}$, leading to $\mathrm{Fr}_{d} \mathrm{We}_{d}=\rho V_{\text {tip }}^{4} / \gamma g$ which compares the effect of inertia upon capillarity and gravity on the jet dynamics, and is, in particular, independent of bubble radius and viscosity.

In order to estimate $G(\mathrm{Mo})=H(\mathrm{Mo}) f(\mathrm{Mo})^{6 / 5}, \mathrm{f}(\mathrm{Mo})$ needs to be known. On the inset of Fig. 18 (a), $\mathrm{We}_{b} \mathrm{Bo}_{b}^{1 / 2}$ is plotted as a function of Mo, as in Fig. 12 (b), but in a log-log plot, allowing us to determine $\mathrm{f}(\mathrm{Mo})$ by fitting the data in the same regime $\left(10^{-11} \lesssim \mathrm{Mo} \lesssim 10^{-8}\right)$. The power law $f(\mathrm{Mo})=\mathcal{B M o}^{2 / 7}$, with $\mathcal{B}=3.910^{4}$, fits reasonably well the experimental data. Consequently, $G(\mathrm{Mo})=$ $\mathcal{A M o}{ }^{-1 / 3}\left(\mathcal{B M o}^{2 / 7}\right)^{6 / 5}=\mathcal{C M o}{ }^{1 / 105} \sim \mathcal{C}$, with $\mathcal{C}=\mathcal{A} \mathcal{B}^{6 / 5}=3.55$. This signifies that 
viscosity is removed from the scaling law relating the drop radius, the jet velocity and the liquid parameters, leading to, for Mo $\lesssim 10^{-8}$ :

$$
\mathrm{Bo}_{d}=\mathcal{C}\left(\mathrm{Fr}_{d} \mathrm{We}_{d}\right)^{-3 / 5} .
$$

On the Fig. 18 (a) the drop Bond number $\mathrm{Bo}_{d}$ is, therefore, plotted as a function of $\mathrm{Fr}_{d} \mathrm{We}_{d}$ and we observe an excellent collapse of all the experimental data represented with closed symbols, confirming Eq. (21). Figure. 18 (b) presents $\mathrm{Bo}_{d} /\left(\operatorname{Fr}_{d} \mathrm{We}_{d}\right)^{-3 / 5}$ as a function of Morton number and confirms that the drop Bond number is independent of viscosity for the closed symbols. This inviscid behaviour stops at Mo $\simeq 10^{-8}$, viscosity playing a role for open symbols, between $10^{-8}$ and $10^{-7}$ (corresponding to $\mu \sim 5$ and $7 \mathrm{mPa}$.s for water glycerol mixtures). Above $\mathrm{Mo} \simeq 10^{-7}$ no more drop can detach.
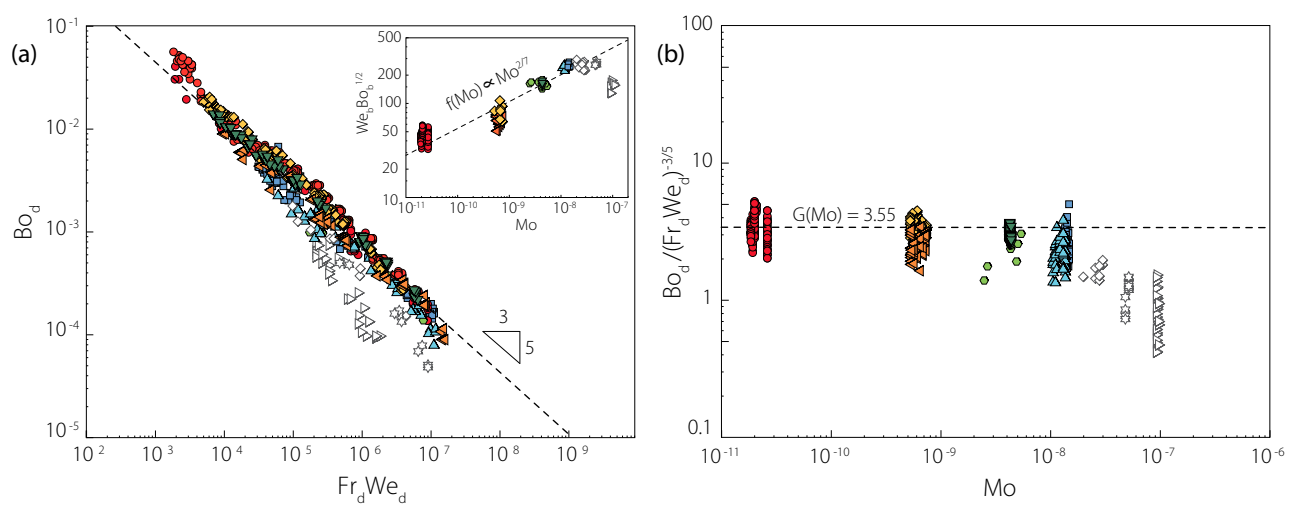

Fig. 18. (a) Drop Bond number as a function of the product of Froud and Weber number $\mathrm{Fr}_{d} \mathrm{We}_{d}=\rho V_{\text {tip }}^{4} / \gamma g$ for various values of the Morton number. For Mo $\lesssim 10^{-8}$, all the data, plotted with closed symbols, collapse on a single curve following the trend $\mathrm{Bo}_{d}=\mathcal{C}\left(\mathrm{Fr}_{d} \mathrm{We}_{d}\right)^{-3 / 5}$ as shown by the dashed line. In the inset, the Fig. 3 (b) of Ghabache et $a .^{7}$ is plotted in a log-log plot, $f(\mathrm{Mo})=\mathcal{B M o}^{2 / 7}$ fits reasonably well the data for Mo $\in\left[10^{-11}, 10^{-8}\right]$ as shown by the dashed line. (b) $\mathrm{Bo}_{d} /\left(\mathrm{Fr}_{d} \mathrm{We}_{d}\right)^{-3 / 5}$ as a function of Morton number. The dashed line fitting the data on the same range is a constant equals to $\mathcal{C}$. Data corresponding to Mo $\in\left[10^{-8}, 10^{-7}\right]$ plotted with open symbols leave the inviscid regime. Above $\mathrm{Mo} \simeq 10^{-7}$ no more drop can detach.

Equation (21), valid for Mo $\lesssim 10^{-8}$, is therefore more robust, with less scattering than Eq. (19). Furthermore, it demonstrates that viscosity does not participate to the drop detachment process. This result was predictable as the Ohnesorge number is always lower than one. However, we may now wonder why the drop radius was dependent on the liquid viscosity in Figs. 9, 17 and Eq. (19). Actually, this influence of viscosity on drop size was only through the jet's formation as a memory of the bubble collapse. Indeed, as we discussed in paragraph 4.4, when a bubble collapses, capillary waves focus at the bottom of the cavity giving birth to the jet. Increasing the liquid viscosity changes the wave focusing, producing a thinner jet and therefore smaller droplets. In Eq. (21), this shaping effect is then entirely contained through $V_{\text {tip }}$ and viscosity can disappear, shedding light on the inviscid behavior of the drop detachment mechanism. Finally, the Bond number of the drop seems to be only selected by a competition between the given inertia, which makes the jet rising and stretching, and the duet gravity-capillarity which pulls on the jet tip so as to form a blob, initiating an end-pinching mechanism and consequently releasing a drop. While 
the influence of capillarity is obvious in this blob formation, the one of gravity can be more surprising. However, at the height the drop is detached, the gravity can already play a role. Indeed, the Froude number built on the drop detachment height $h_{\text {det }}$, $\operatorname{Fr} h_{\text {det }}=V_{\text {tip }}^{2} / g h_{\text {det }}$, equals to $\mathrm{O}(1)$ for top drops projected by the largest bubbles.

\subsection{Conclusion and applications}

Here we have provided experimentally two different scaling laws giving the top jet drop radius ejected after a bubble burst as a function of the liquid parameters and the mother bubble radius in Eq. (19) or the jet velocity in Eq. (21). These results induce various outcomes. The size distribution of the top jet drop aerosol can now easily be computed as long as we know the bubble size distribution, which is the case in ocean for example $55 ; 56$. These results also apply to slightly viscous liquids (up to Mo $\sim 10^{-8}$ ) like champagne or sparkling wine obviously ${ }^{30}$.

Furthermore, these two scaling laws enable to untangle the intricate role of viscosity in the end pinching mechanism by defining exactly at which step of the bubble bursting process it influences the drop size selection. Indeed, viscosity appears in Eq. (19) and not in Eq. (21), namely when the drop size is expressed as a function of the bubble radius and not when it is expressed as a function of the jet velocity. Moreover, we saw that, when a bubble collapses, it generates a jet whose velocity is selected by various ingredient including viscosity (see section 4). Therefore, viscosity appears in Eq. (19) because of its role on the jet velocity selection and, once the jet is rising, viscosity does not play an active role anymore, in particular in the drop detachment, and stays hidden in the jet velocity in the Eq. (21). This implies that, once the jet velocity and shape are given, inviscid considerations would properly describe the drop detachment. Finally, contrary to the liquid viscosity which does not participate to the drop detachment process itself $\left(\mathrm{Mo} \lesssim 10^{-8}\right)$, the duet gravity-capillarity seems to initiate the drop detachment by balancing the jet inertia and pulling on the jet tip.

\section{Evaporation of droplets in a Champagne wine aerosol}

In the two last sections, we have characterized in details, and in a general context, the effervescence mechanism from the bubble lying at the free surface up to the drop detachment. In particular, we have showed that champagne aerosol is in a zone of control parameters where a slight change of liquid properties leads to a drastic change of the drop velocity and size produced by bubble bursting. In the present section, we will focus on champagne properties and, driven by the knowledge we have gained in the study of jet and drop detachment dynamics, we now aim at estimating droplet evaporation and finding if liquid properties would enable to optimize the aerosol evaporation. This section is extracted from Ghabache et al. $(2016)^{58}$.

\subsection{Top drop velocity and size}

For the sake of clarity we keep restricting our attention on the droplets that bound the edge of the aerosol cloud. Indeed, we will see in paragraph 6.6 that they dominate the evaporation process as they are faster and with a size at least comparable if it is not bigger than the others (see Fig. 4 or 14 for instance). In particular, we will see that our conclusions based on the highest drops would not be modified by taking into account the other drops. 

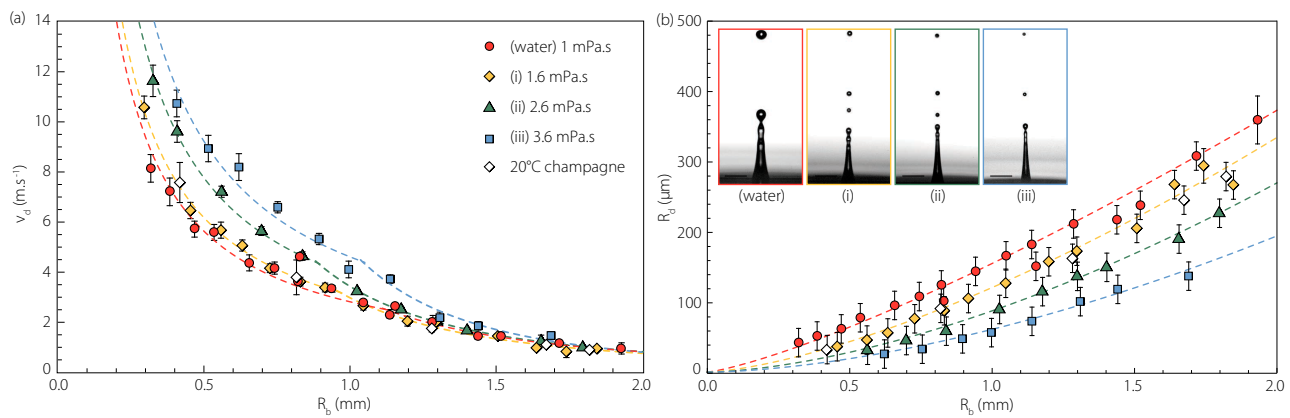

Fig. 19. (a) Top drop velocity $V_{d}$ as a function of the bubble radius $R_{b}$ in water, champagne at $20^{\circ} \mathrm{C}$ and in the three hydro-alcoholic solutions (i)-(iii). The dashed line curves are the best fits of the experimental values of the drop velocity. (b) Top drop radius as a function of bubble radius in water, champagne and in the three hydro-alcoholic solutions (i)-(iii). The dashed lines are the best fits of the four series of data. The four snapshots display the jet and jet drops for each solution.

We set out by plotting in Fig. 19 (a) the dependence of the top drop velocity $V_{d}$ on mother bubble radius $R_{b}$ in water, champagne and the three hydro-alcoholic solutions (i)-(iii) presented in Table 1. We recall that $V_{d}$ is measured when the drop detaches and the three solutions (i)-(iii) have the same properties as a typical champagne at three different temperatures, respectively 20,12 and $4^{\circ} \mathrm{C}$. Fig. 19 (b) presents the top drop radius as a function of the bubble radius $R_{b}$ from our experiments in our five different liquids. The four snapshots display the jet and jet drops for each solution. As we showed in the previous sections, the drop velocity is greater and the top drop is shrinking as the bubble radius is smaller and the liquid is more viscous. In the two graphs, the dashed lines are the best fit of the experimental drop velocity and radius as a function of the bubble radius. They will be used later on as initial drop radius and velocity in the drop dynamics differential equations. Finally, the superposition of the highest jet drop velocity in champagne and in the water-ethanol solution (i) is excellent (see diamonds), confirming that those solutions perfect idealized champagne and confirming that surfactants can be neglected in the jet drop dynamics.

\subsection{Maximum drop height}

The top drop maximum height is plotted on Fig. 20 as a function of the bubble radius from our experiments in distilled water and for the three solutions (i)-(iii). The points in water are in good agreement with the literature ${ }^{37 ; 59}$. All these experimental data adopt a bell shape curve with a maximum height reached for bubble radius around one millimeter. The maximum height decreases and is slightly shifted toward larger radius as viscosity is increased. This diminution of the maximum height with viscosity is surprising as we saw that increasing viscosity leads to faster drop ejection velocity. However, because this increase in drop velocity is accompanied by a size shrinking, the effect of drop inertia (which scales with the drop volume), should be compared with drag force $\mathrm{F}_{D}$ (roughly speaking scaling as a surface). When drop size decreases, surface effects become dominant over volume effects and consequently, small drop inertia becomes negligible compared to the drag force. The maximum height a drop can reach with a given initial velocity can thus be captured by integrating the following differential equation : 


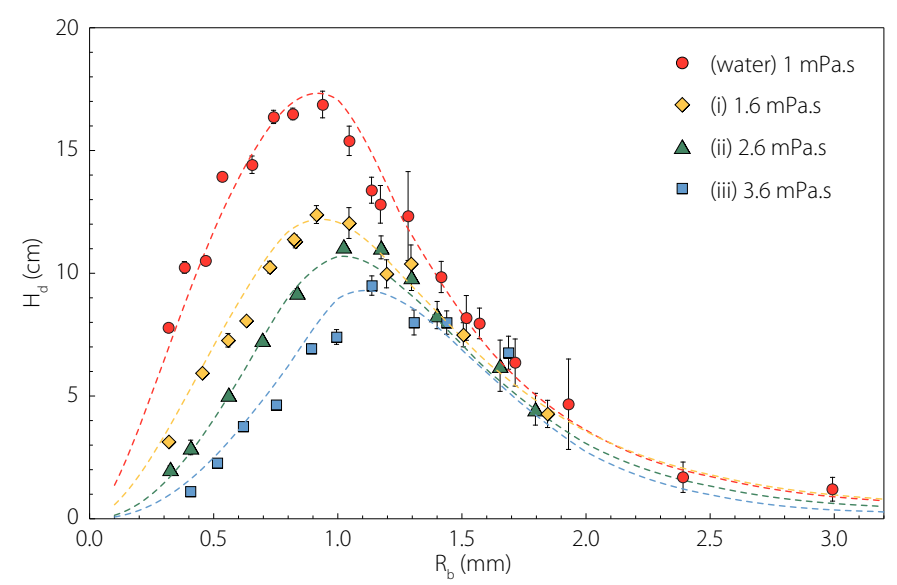

Fig. 20. Top drop maximum height as a function of bubble radius in water and for the three hydro-alcoholic solutions (i)-(iii). The dashed line curves are computed numerically by integrating the Newton's second law applied to the flying drop using drag coefficient $C_{D}=\frac{24}{\operatorname{Re}(t)}\left(1+0.15 \operatorname{Re}(t)^{0.687}\right)$, experimental drop radius $R_{d}\left(R_{b}\right)$ and initial drop velocity $V_{d}\left(R_{b}\right)$ as initial condition.

$$
\frac{4}{3} \rho \pi R_{d}^{3} \frac{d^{2} z}{d t^{2}}=-\frac{1}{2} \rho_{\text {air }} \pi R_{d}^{2}\left(\frac{d z}{d t}\right)^{2} C_{D}-\frac{4}{3} \rho \pi R_{d}^{3} g
$$

with $z$ the drop height and $C_{D}$ the drop drag coefficient. Here $C_{D}$ is taken as the drag coefficient on a solid sphere in steady motion. As the drop Reynolds number $\operatorname{Re}(t)=2 \frac{\rho_{\text {air }}}{\mu_{\text {air }}} \frac{d z}{d t} R_{d}$ ranges from ten to a hundred, classical Stokes' drag $24 / \operatorname{Re}$ is not adequate and inertial terms need to be added. Many empirical or semiempirical equations have been proposed ${ }^{60}$ to approximate $C_{D}$ as a function of the Reynolds number on a given Reynolds range. Here $C_{D}=\frac{24}{\operatorname{Re}(t)}\left(1+0.15 \operatorname{Re}(t)^{0.687}\right)$ valid for $R e<800^{61}$, has been taken. On Fig. 20 we plot with a colored dashed line the top drop height as a function of the bubble radius computed with this model without any fitting parameters. We observe an excellent agreement between these curves and the experimental points. The drop maximum height is perfectly captured by this simple model containing the experimental drop radius $R_{d}\left(R_{b}\right)$ and the initial drop velocity $V_{d}\left(R_{b}\right)$ as initial condition. It also confirms the validity of the strong hypotheses of steady drag and drop sphere shape.

\subsection{Simple model for drop evaporation}

In this system, drop evaporation is crucial to understand the aroma diffusion by champagne aerosol. We have all the informations needed to build a simple predictive model, and to highlight the relevant features playing a role in the evaporation dynamics. Hence, a single droplet with radius $R_{d}(t)$, velocity $\frac{\mathrm{d} z}{\mathrm{~d} t}$ and temperature $T$ (between 4 and $20^{\circ} \mathrm{C}$ ) is moving into an environment with temperature $T_{\infty}=20^{\circ} \mathrm{C}$ and mass fraction of the vapor of the droplet material $Y_{\mathrm{vap}}^{\infty}=\frac{\rho_{\mathrm{vap}}^{\infty}}{\rho_{\text {air }}}$. The evaporation process of the droplet depends a priori on these parameters. The considered model implies that quasi-steady conditions prevail. Under these conditions, and considering the thermophysical properties as constant, the analysis of mass transfer processes into 
the gas phase near the droplet surface allows the determination of the regression rate of the droplet radius :

$$
\frac{\mathrm{d} R_{d}^{2}(t)}{\mathrm{d} t}=-2 j_{0}\left(1+0.3 \mathrm{Sc}^{\frac{1}{3}} \operatorname{Re}(t)^{\frac{1}{2}}\right)
$$

where Sc, the Schmidt number, is defined as the ratio of momentum diffusivity in air $\left(\nu_{\text {air }}\right)$ and mass diffusivity of vapor in air $(D)$, and $j_{0}$ the evaporation parameter ${ }^{62 ; 63}$. In our case, where the gas temperature is low, evaporation process is only controlled by diffusion, which leads to $j_{0}=\frac{\rho_{\text {air }}}{\rho_{\text {liq }}} D\left(Y_{\text {vap }}^{\text {surf }}-Y_{\text {vap }}^{\infty}\right)^{64}$ - the Stefan flow being negligible $^{63}$. For the calculation of the vapor mass fraction of each substance at the surface of the droplet $Y_{\text {vap }}^{\text {surf }}$, the heat transfer equation and the Clausius-Clapeyron equation are considered. Because the vapor pressure of the glycerol is about six orders of magnitude lower than this of water or ethanol, evaporation of glycerol has been neglected. With the intrinsic difference of volatility between water and ethanol, and an ambient partial pressure taken to $50 \%$ of vapor pressure for water (relative humidity) and $0 \%$ for ethanol, we obtain an evaporation parameter $\left(j_{0}\right)$ for ethanol around five times greater than evaporation parameter for water. Expectedly, ethanol evaporates easier than water. More details on this analysis are given in the "Methods" section. Equation (23) is the product of two terms : the well known $d^{2}$-law for evaporation of an unmoving droplet which follows from equation $\frac{\mathrm{d} R_{d}^{2}(t)}{\mathrm{d} t}=-2 j_{0}{ }^{65 ; 66}$. And, the drop motion which is taken into account using the standard Ranz and Marshall empirical mass transfer correlations for moving sphere $1+0.3 \mathrm{Sc}^{\frac{1}{3}} \operatorname{Re}(t)^{\frac{1}{2}} 67$. This correction comes from the assumption that the mass exchange between the droplet surface and the gas may be modeled as occurring within a spherical diffusion film of constant thickness $R_{d}(t) /\left(0.3 \mathrm{Sc}^{\frac{1}{3}} \operatorname{Re}(t)^{\frac{1}{2}}\right)$ - this behavior goes by the names of Prandlt-Blasius-Pohlhausen $(1921)^{68}$.

\subsection{Drop evaporation from trajectory}

Now, the system of differential equations (Eqs. (22) \& (23)) can be solved, with the variable $R_{d}(\mathrm{t})$ instead of the constant $R_{d}$ in Eq. (22). The initial conditions $\dot{z}(0)$ and $R_{d}(0)$ are given by the experimental measurement of the initial drop velocity and radius, resp. $V_{d}$ and $R_{d}$ (Fig. 19). On the Fig. 21(a) the experimental height of the first jet drop ejected after a bubble burst is plotted as a function of time with grey circles in the case of a small drop. As expected, the curve is typical of a trajectory where drag forces prevail over inertia. On the same graph, the theoretical trajectories, without evaporation (Eq. (22)), and with evaporation (Eqs. (22) \& (23)) are plotted respectively with dashed black and plain red curves. Because the determination of the initial radius $R_{d}$ is not very accurate $\left(R_{d}=24 \pm 3 \mu \mathrm{m}\right)$, the error bar needs to be included in the model. In this case, the hatched gray zone and the red zone represent the error bar in the numerical resolution induced by the experimental error on the initial drop size measurement. Despite the errors and approximations, the experimental trajectory is very well captured by our evaporation model and could not be captured with just a constant radius trajectory model (Eq. (22)). Our evaporation model is therefore well adapted to this system and will be used in the following in order to estimate the evaporated liquid mass during the drop time of flight. The discrepancy at the end of the trajectory may be due to an experimental gradient of humidity getting closer to $100 \%$ as $z$ gets to 0 (close to the free surface).

Figure $21(\mathrm{c})$ presents the radius variation $\left(\delta R_{d} / R_{d}(0)\right)$ of the same drop as in Fig. 21(a) on its time of flight. We observe an almost constant radius shrinking rate during the drop free fall, reaching a final value of about $20 \%$ before landing again. 

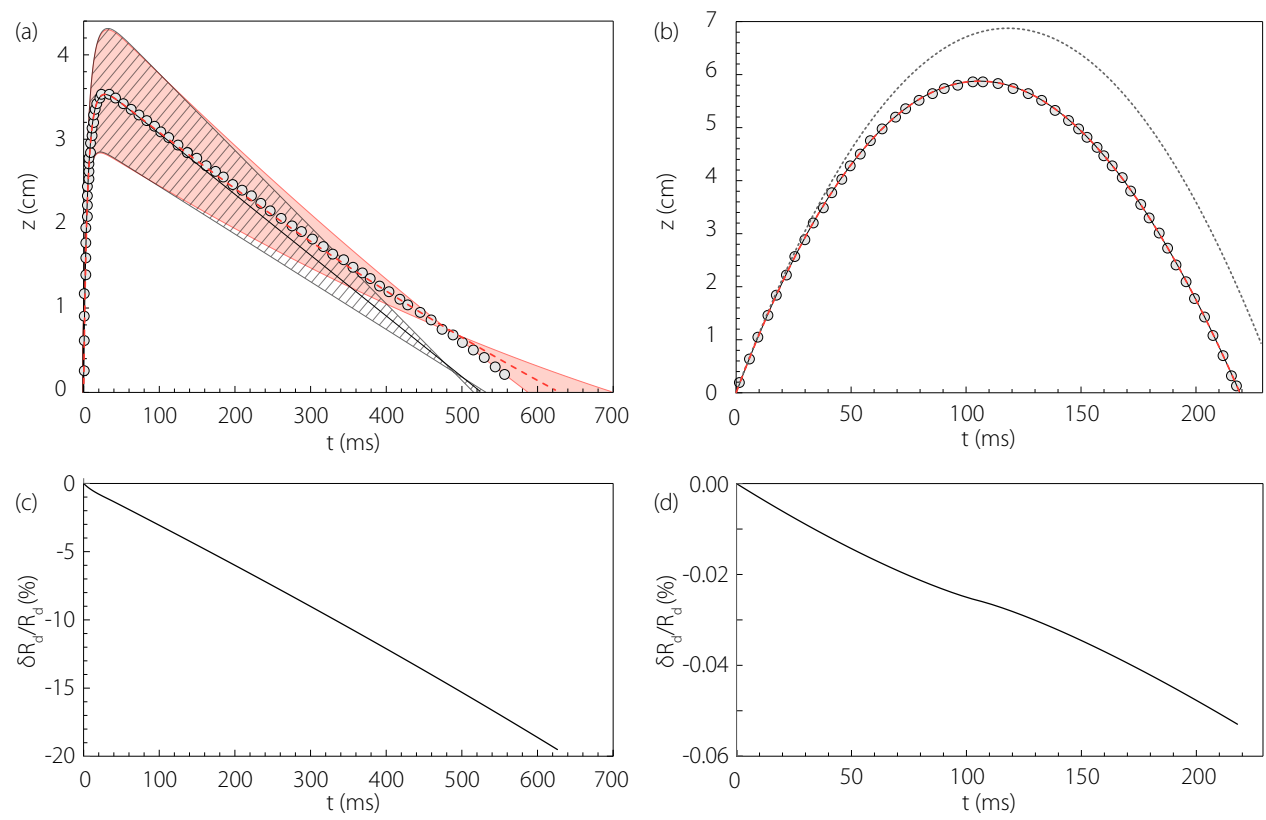

Fig. 21. (a) Drop height $z$ as a function of time for a typical small droplet : $R_{d}=24$ $\mu \mathrm{m}$ and $V_{d}=8.30 \mathrm{~m} . \mathrm{s}^{-1}$. The gray circles correspond to the experimental trajectory, the dashed black curve corresponds to the trajectory obtained by solving numerically Eq. (22) (no evaporation, $R_{d}$ constant) and the plain red curve corresponds to the trajectory obtained by solving numerically Eqs. (22) \& (23) (with evaporation, $R_{d}(\mathrm{t})$ non constant). The hatched gray and red zones represent the error in the numerical resolution of the respective models (22) and (22) \& (23) induced by the experimental error on the initial drop size measurement : $R_{d}=24 \pm 3 \mu \mathrm{m}$ (experimental error on the initial velocity is negligible). (b) Drop height $z$ as a function of time for a typical big drop : $R_{d}=307 \mu \mathrm{m}$ and $V_{d}=1.16 \mathrm{~m} . \mathrm{s}^{-1}$. In this case the experimental trajectory (gray circles) and the numerical trajectory with evaporation (plain red) and without evaporation (dashed black) all collapse. The dotted curve corresponds to the ballistic trajectory $z(t)=V_{d} t-\frac{1}{2} g t^{2}$. (c) Drop radius variation rate $\left(\delta R_{d}(t) / R_{d}(0)\right.$, with $\left.\delta R_{d}=R_{d}(0)-R_{d}(t)\right)$ for the drop corresponding to Fig.(a) $\left(R_{d}(0)=24 \mu \mathrm{m}\right)$, on its time of flight. Here $\delta R_{d}(t) / R_{d}(0)$ reaches a final value of about $20 \%$ before landing again. (d) Drop radius variation rate for the drop corresponding to Fig.(b) $\left(R_{d}(0)=307 \mu \mathrm{m}\right)$, on its time of flight. Here $\delta R_{d}(t) / R_{d}(0)$ reaches a final value less than $0.06 \%$ before landing again.

Obviously, small droplets trajectory are more prone to being affected by evaporation. This is why, on Fig. 21(b), the experimental trajectory of a drop among our biggest $\left(R_{d}=307 \mu \mathrm{m}\right)$, collapses with the two theoretical trajectories. Evaporation does not modify its trajectory. Indeed, in this case $\delta R_{d} / R_{d}(0)$ reaches less than $0.06 \%$ as shown on Fig. (d). Note that, the "plateau" observed on the drop shrinking rate, corresponds to the time when the drop stopped at its maximum height, showing the influence of the drop motion on its evaporation. Finally, on Fig. 21(b) the purely ballistic trajectory without drag has been added with a grey dotted line. We observe that, as the drop size increases, the influence of the drag becomes less significant and the trajectory approximates to a parabola. 


\subsection{Discussion on the top drop evaporation}

Fig. 22 presents the bubble radius dependence of the total mass evaporated on the top drop trajectory. Namely, $\delta M=\frac{4}{3} \pi \rho_{\text {liq }}\left(R_{d}^{3}(0)-R_{d}^{3}\left(\mathcal{T}_{\text {fly }}\right)\right)$ with $\mathcal{T}_{\text {fly }}$ the drop time of flight. The only differences in the calculation of the three curves (i)-(iii) are the initial conditions $\dot{z}(0)=V_{d}\left(R_{b}\right)$ and $R_{d}(0)=R_{d}\left(R_{b}\right)$ that change because of viscosity (see Fig. 19). The first interesting result is the absolute value of these curves, around 0.1 $\mu \mathrm{g}$ per drop. Considering that approximately between 300 and 500 bubbles burst per second at the surface of a champagne glass ${ }^{1}$, one obtains an approximative value of the top drops evaporation rate $: \dot{M}=40 \mu \mathrm{g} . \mathrm{s}^{-1}$. This value needs to be compared to the evaporation rate from the flat surface $\left(\mathcal{S}_{l}\right)$ of a glass filled with the same solution. In this aim, the evaporation flux from the surface is taken as purely diffusive and integrated on the length scale of the vapor concentration gradient $\mathcal{L}_{\infty}{ }^{69}$. This gives the evaporation rate from the surface $: \dot{m}=-\frac{D \mathcal{S}_{l} \rho_{\text {air }}}{\mathcal{L}_{\infty}}\left(Y_{\text {vap }}^{\text {surf }}-Y_{\text {vap }}^{\infty}\right)$. In this context $\mathcal{L}_{\infty}$ is typically the height between the liquid surface and the top of the glass. In a flute the interfacial area $\mathcal{S}_{l}$ is a disc of approximate radius two centimeters and $\mathcal{L}_{\infty}$ is taken equal to the flute diameter: four centimeters. The other values are the same as for the drop evaporation. In this case, $\frac{\dot{M}}{\dot{m}} \simeq 10$, which means that the aerosol constituted by the top drops evaporate ten times more than the still liquid surface. This key result confirms, for the first time, the universal feeling that the characteristic fizz of a sparkling wine is of a paramount importance in the flavor release. Now, let us look whether evaporation of this aerosol can be optimized.

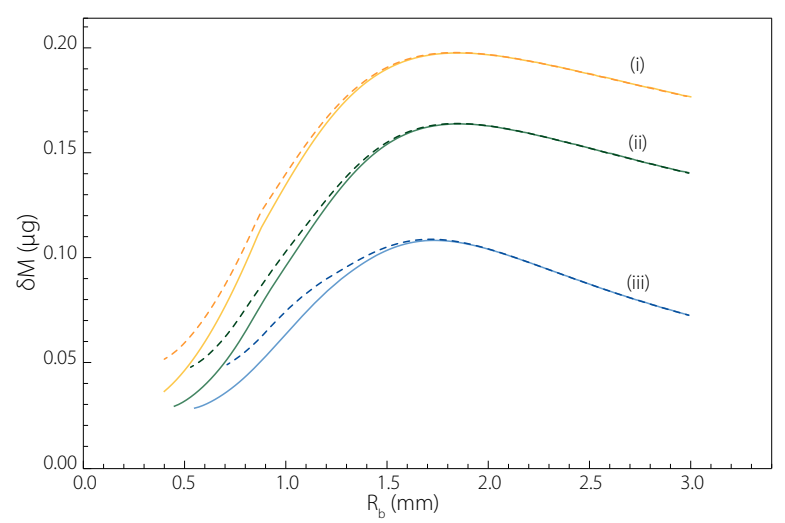

Fig. 22. The plain lines present the total evaporated mass $\delta \mathrm{M}$ from the top drop during its time of flight $\mathcal{T}_{\text {fly }}$, computed numerically with Eqs. (22) and (23), as a function of the bubble radius $R_{b}$ in the three solutions (i)-(iii). For the calculation, ambient relative humidity above the tank has been taken to $50 \%$. The dashed lines present the approximated evaporated mass Eq. (24) in the case where $R_{d}(t) \simeq R_{d}(0)$ (initial drop radius) with a parabolic trajectory. The approximation is satisfactory for a high part of the range of bubble radius.

On Fig. 22 each curve has a bell shape, which means that there is a bubble radius optimizing the top drop evaporation. The liquid viscosity also plays a role, the evaporation of the top drop is more efficient if the bubble bursting takes place in a weakly viscous liquid. One observes that, the maximums occur roughly for the same bubble radius. Note that this latter does not correspond either to the maximum drop height, the maximum drop velocity (smallest bubbles) or the maximum drop radius (largest bubbles). However, these particular bubbles (with $R_{b} \sim 1.7-1.8 \mathrm{~mm}$ ) which would optimize the aroma diffusion in the context of champagne tasting, are 
quite large. In the popular mind, it is nevertheless often said that the smaller the bubbles, the better the champagne; our results undermine this popular belief, at least in terms of sense of smell. Especially considering that, both the size of bubbles and their nucleation rate are mainly driven by the level of dissolved $\mathrm{CO}_{2}{ }^{70}$. Indeed, this means that champagnes poor in dissolved $\mathrm{CO}_{2}$ (typically old ones) will combine small bubbles and low nucleation rate inducing systematically less numerous bubbles. On the other hand, champagne rich in dissolved $\mathrm{CO}_{2}$, combining large bubbles and high bubble nucleation rates will show a much better efficiency in terms of flavor release.

In order to interpret these curves we need to identify the relevant ingredients that influence the evaporation of these jet droplets. To this end, let us consider the limit case where the drop is big enough to allow the trajectory to be approximated by a parabola : $z(t)=V_{d} t-\frac{1}{2} g t^{2}$ and $V_{d}=g \mathcal{T}_{\text {fly }} / 2$. In this case, evaporation does not significantly affect the drop radius : $R_{d}(t) \simeq R_{d}(0)$ (inset of Fig. 21). Eq. (23) can easily be integrated and the total evaporated mass can be approximated by :

$$
\delta M=2 \pi \rho_{\text {liq }} j_{0} \mathrm{R}_{d} \mathcal{T}_{\text {fly }}\left(1+0.3 \mathrm{Sc}^{\frac{1}{3}}\left(\frac{4 g}{9 \nu_{\text {air }}}\right)^{\frac{1}{2}}\left(\mathrm{R}_{d} \mathcal{T}_{\text {fly }}\right)^{\frac{1}{2}}\right)
$$

This approximated development of the evaporated mass is plotted on Fig. 22 with dashed line. For the smaller drops, Eq. (24) slightly overestimates the evaporated mass, because in this case the drop trajectory is far from being parabolic and a drop can significantly shrink during its time of flight. However, the agreement is perfect for the bigger drops, including the maximum, particularly well captured. In first approximation, the trajectory of a drop ejected by a bursting bubble is thus relatively well approximated by a parabola. This allows us to identify the product $R_{d} \mathcal{T}_{\text {fly }}$ as the relevant quantity for drop evaporation in this system. The first term comes from the evaporation of an unmoving drop of radius $R_{d}$ in air during the time $\mathcal{T}_{\text {fly }}$. The second term comes from the drop motion. The two terms have the same order of magnitude. $R_{d}$ and $\mathcal{T}_{\text {fly }}$ contain all the ingredients needed to estimate the first jet drop evaporated mass.

\subsection{Evaporation of the second drop}

Here we have focused on the evaporation of the first drop detached from the bubble bursting jet. We can now evaluate the evaporation of the second drop to look at its influence on the total evaporation of a bubble bursting aerosol. We solve the Eqs. (22) and (23) with, as initial conditions, the second drop radius and velocity measured experimentally. Here, we still consider that the drop is isolated and that its evaporation is not influenced by the top drops. Typically we take the same ambient relative humidity $(50 \%)$ and concentration of vapor of ethanol $(0 \%)$ as for the first drop. Consequently, we probably slightly overestimate the evaporation of the second drop.

On Fig. 23, the evaporated mass $\delta \mathrm{M}$ from the top drop (plain line), the second drop (small dashed line) and the sum of both (long dashed line) is plotted as a function of the bubble radius $R_{b}$ in the three solutions (i)-(iii). The evaporation of the first drop clearly prevails over the evaporation of the second drop, especially considering that here the evaporation of the second drop might be overestimated. Typically, the evaporation of the second drop does not modify the bubble radius that optimizes the first drop evaporation. Therefore, our conclusions based on the first drop are not fundamentally modified by taking into account the second drop, and most probably the third drop, etc. This can be explained by the fact hat the first drop is faster and usually bigger than the others or with a comparable size. Obviously, if this first work 

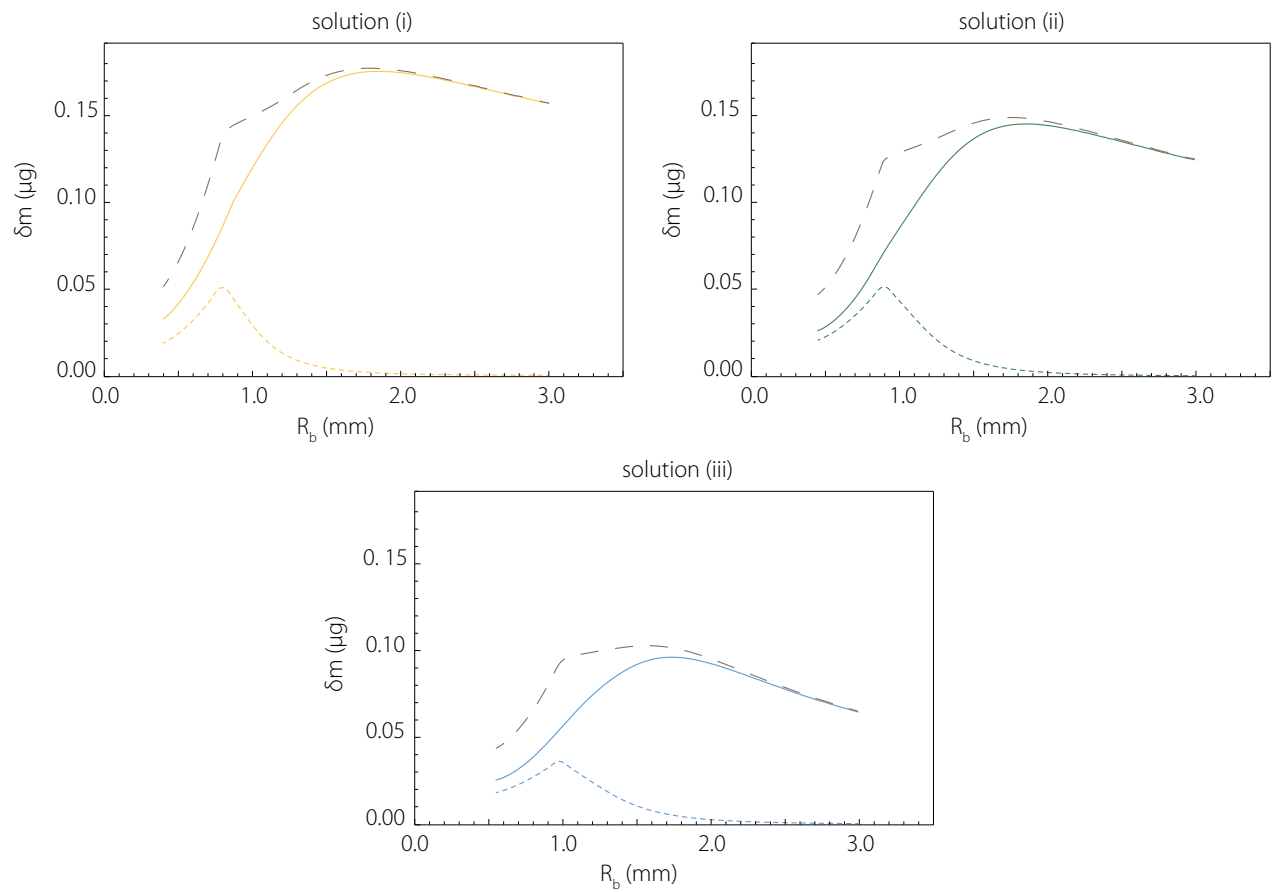

Fig. 23. Total evaporated mass $\delta \mathrm{M}$ from the top drop (plain line) and second drop (small dashed line) during their time of flight, computed numerically with Eqs. (22) and (23), is plotted as a function of the bubble radius $R_{b}$ in the three solutions (i)-(iii). The sum of evaporated mass of top and second drops are plotted with the long dashed line. For each case the contribution of the second drop is small compared to the contribution of the top drop. In a bubble bursting aerosol the evaporation of the top drops is dominant.

is aimed at giving tendencies, it does not pretend to be accurate and further studies need to be realized in order to know the amount of liquid evaporated above a glass of champagne in tasting conditions.

\section{Conclusion}

Finally, even though our work is based on a model experiment, it presents unique contributions on the bursting bubble dynamics, from the bubble lying at the free surface to the evaporation of the produced droplets. The outcomes are important both from a fundamental point of view and in the application to champagne aroma diffusion.

In the general context of the physics of effervescence, we provided a comprehensive picture of the entire process : bubble collapse, jet dynamics, top drop detachment and trajectories. In all cases, we interpreted the physical mechanisms at play and we provided scaling laws based on experimental results. In particular, the top drop velocity and size are given as a function of bubble size and liquid properties on very large ranges of the relevant non-dimensional numbers.

Afterward, we showed that the range of liquid parameters of Champagne wines takes place in the most appealing zone of the space phase, where a slight change of liquid properties leads to a drastic change of the drop velocity and size produced by bubble bursting. Furthermore, we proved experimentally that the aerosol above 
hydro-alcoholic solutions like champagne or any sparkling wine is only constituted by jet drops, no film drops populate such aerosol. It is therefore very different from sea spray where film drops are crucial and represents a genuine academic aerosol.

Based on this observation, we proved that hydro-alcoholic solutions perfectly mimic champagne in terms of aerosol production and can be used as a model champagne. The role of the surfactants present in champagne is thus negligible in the drop dynamics. Finally, we demonstrated that this aerosol plays a critical role in the aroma diffusion. Indeed, compared to a still wine, it drastically improves the transfer of liquid into the surrounding air, even though it is only populated by jet drops. We exhibited conditions on bubble bursting that optimize aerosol evaporation : large bubbles and weakly viscous liquids. We identified a large bubble radius $(\sim 1.7 \mathrm{~mm})$, broadly common to the whole range of champagne viscosity, that makes liquid transfer more efficient. This could be easily achievable as laser-etching on champagne glasses allows the creation of monodisperse bubbles reaching the surface at a chosen radius ${ }^{57}$. This result is also remarkable as it undermines the popular belief that the smaller the bubbles, the better the champagne. Small bubbles being the worst in terms of aroma release. We also showed that decreasing champagne viscosity would improve drop evaporation. In this aim, additives that would change wine viscosity without changing the taste might be used. These results pave therefore the way towards the fine tuning of champagne aroma diffusion, a major issue for the sparkling wine industry, and should encourage further research on this subject. In particular, around the collective effects of bubble raft on the aerosol production, last major step toward a truly quantitative relevancy of academic results for applications to champagne aroma diffusion in tasting condition.

\section{Bubbles raft and collective effects}

As introduced just above, most of our results have been obtained with a single bubble bursting at a calm surface. However, as we all know, bubbles rising after a wave breaks in the ocean or in a glass of a sparkling beverage are numerous. In this section we present the existing results, mostly qualitative, on the collective effects of bubbles bursting at the free surface in the presence of neighbor bubbles.

\subsection{When champagne bubbles dress up like flowers}

A few seconds after pouring, and after the collapse of the foamy head, the surface of a champagne flute is covered with a layer of mono-disperse bubbles - a kind of bubble raft, or 2D foam, where each bubble is generally surrounded by six neighboring bubbles (see Fig. 24). Bubbles arrange themselves in an approximate hexagonal pattern.

In this configuration, when the bubble-cap of a bubble ruptures and leaves an open cavity at the free surface, adjacent bubble-caps are sucked towards this empty cavity and create unexpected and short-lived flower-shaped structures, unfortunately invisible to the naked-eye (see top images of Fig. 25) ${ }^{71 ; 29}$. Shear stresses undergone by bubbles trapped in the close vicinity of a collapsing one are clearly visualized on the bottom image of Fig. 25, where the bubble raft is not complete. Adjacent bubble-caps are sucked and not blown-up by bursting bubbles, contrary to what could have been expected at first glance.

Actually, after the disintegration of a bubble-cap, the hexagonal symmetry around adjoining bubbles is suddenly locally broken. Therefore, the symmetry in the field of capillary pressure around adjoining bubbles is also locally broken. Capillary pressure 


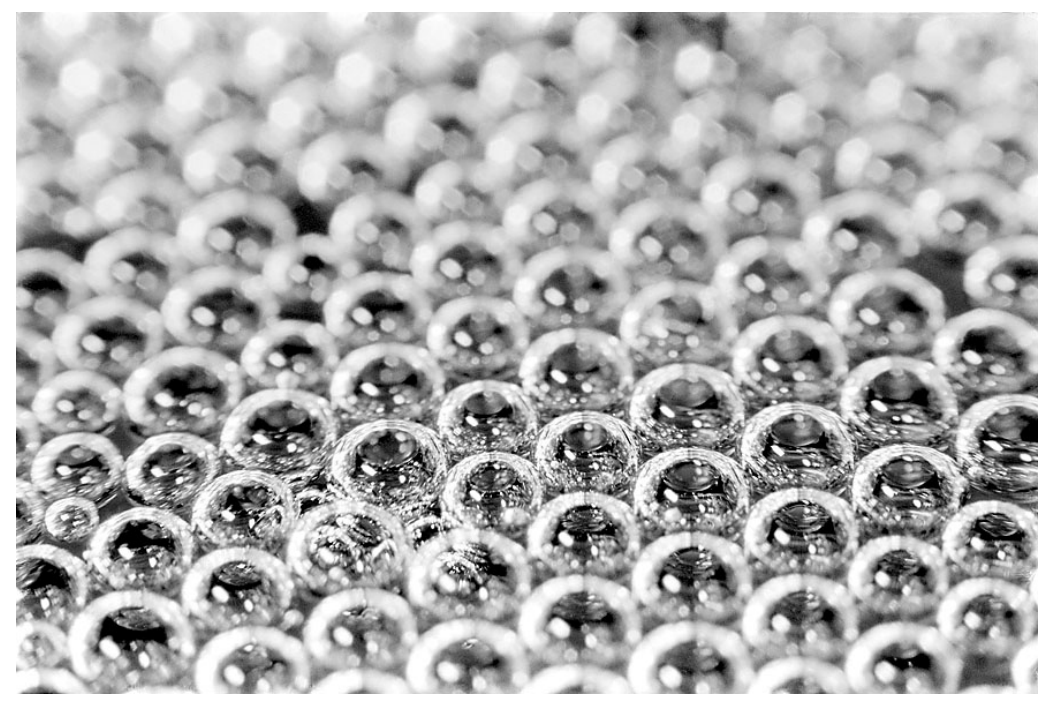

Fig. 24. A few seconds after pouring, and after the collapse of the foamy head, the surface of a flute is covered with a layer of quite mono disperse millimetric bubbles, where bubbles arrange themselves in an approximate hexagonal pattern, strikingly resembling those in beeswax (photograph by Gérard Liger-Belair).

gradients all around the now empty cavity. Indeed, the pressure in the bubble-cap $P_{\text {cap }}$ can be expressed as $P_{\text {cap }}=P_{\text {atm }}+2 \gamma / R_{\text {cap }}=P_{\text {atm }}+\gamma / R_{b}$ (see Fig. 26) with $R_{\text {cap }}=2 R_{b}$ (see Eq. 5). On the other hand, pressure under the open cavity $P_{\mathrm{c}}$ is simply $P_{\mathrm{c}}=P_{\mathrm{atm}}-2 \gamma / R_{b}$. Consequently, the capillary pressure gradient in the thin liquid film of adjoining bubble-caps can be estimated as :

$$
\operatorname{grad} P \sim \frac{P_{\mathrm{c}}-P_{\text {cap }}}{R_{b}}=-\frac{3 \gamma}{R_{b}^{2}}
$$

This capillary pressure gradient in the bubble-caps adjacent to an empty cavity is supposed to be the main driving force of the violent sucking process experienced by a bubble-cap in touch with a bursting bubble. More recently, those flower-shaped structures have been observed during the coarsening of bi-dimensional aqueous foams, obtained by mixing a surfactant, sodium dodecyl sulphate (SDS), with pure water ${ }^{73}$.

During this sudden stretching process, adjacent bubble-caps areas significantly increase. A systematic image analysis of numerous sequences similar to that displayed in Fig. 27 demonstrated an average increase $\Delta \mathrm{A}$ around $15 \%$, of bubble-caps areas adjacent to a central collapsing bubble. The free energy of such a system will be supposed to be mainly stored as surface free energy. Consequently, the density of free energy per unit of volume in the liquid film of a distorted bubble-cap (one petal of the flower-shaped structure) can be evaluated as follows ${ }^{71}$,

$$
\left(\frac{\Delta E}{V}\right)_{\text {bubble-cap }} \sim \frac{2 \gamma}{e} \frac{\Delta A}{A} \sim 0.15 \times \frac{2 \gamma}{e} \simeq 10^{4}-10^{5} \mathrm{~J} \cdot \mathrm{m}^{-3}=10^{4}-10^{5} \mathrm{~N} \cdot \mathrm{m}^{-2}
$$

where $\Delta \mathrm{E}$ is the corresponding surface free energy in excess during the stretching process, $V$ is the volume of the thin liquid film of the emerging bubble-cap, $A$ is the emerging bubble-cap area, and $e$ is the thickness of the thin liquid film of the bubble-cap (of order of $10^{-6}-10^{-7} \mathrm{~m}$ ). Therefore, the liquid flows induced by the 

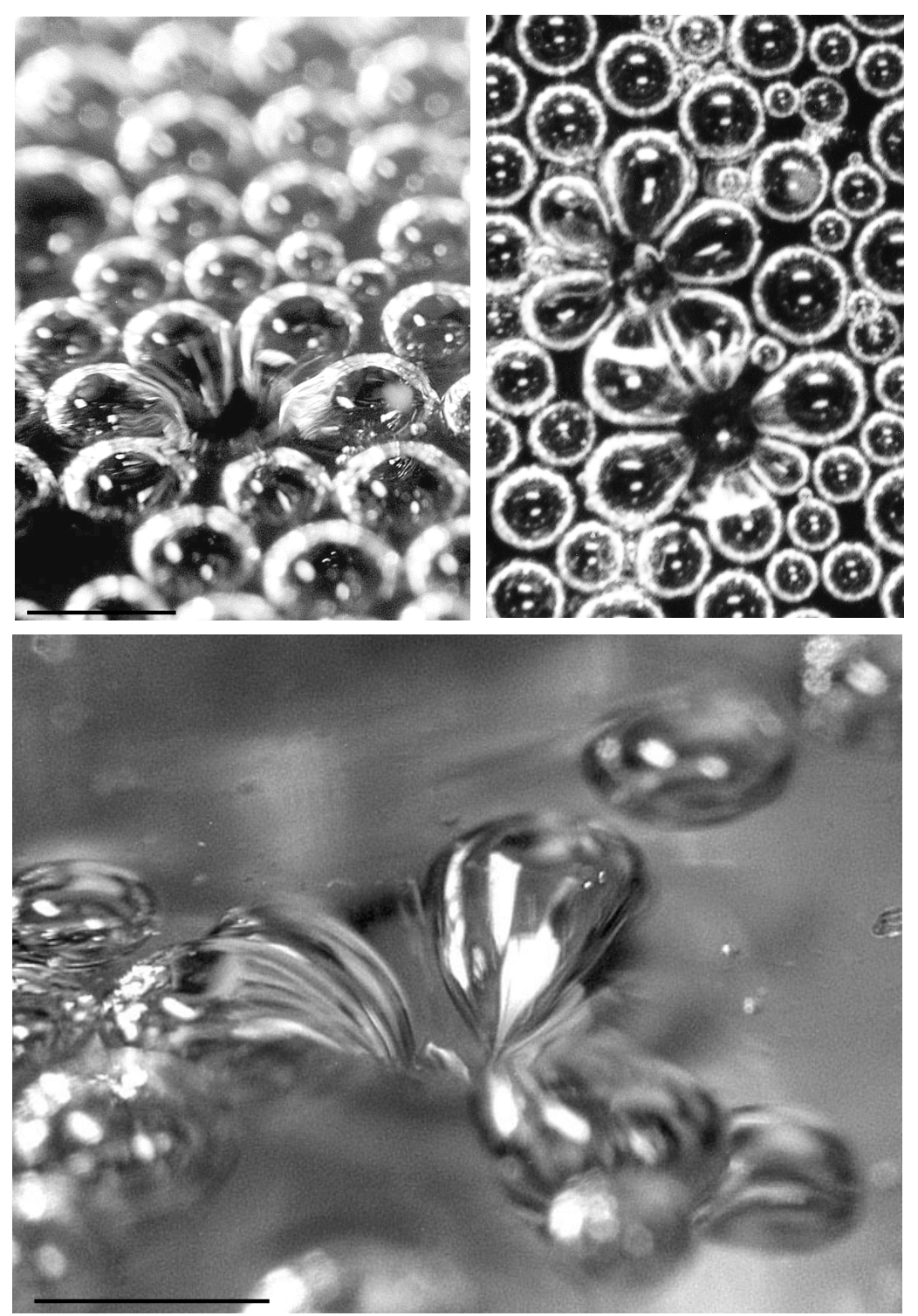

Fig. 25. Top images : Flower-shaped structure, as frozen through high-speed photography, found during the collapse of bubbles in the bubble raft at the free surface of a flute poured with champagne. Bottom image : Shear stresses experienced by bubbles adjacent to a collapsing one at the free surface of a flute poured with champagne. In both cases bar $=1 \mathrm{~mm}$. (Photographs by Gérard Liger-Belair).

capillary pressure gradients are responsible for a density of energy per unit of volume dimensionally equivalent to shear stresses of order of $10^{4}-10^{5} \mathrm{~N} \cdot \mathrm{m}^{-2}$ (depending on the film thickness). By comparison, in previous studies, numerical models conducted to stresses of order of (only) $10^{3} \mathrm{~N} . \mathrm{m}^{-2}$ in the boundary layer around single millimetric collapsing bubbles ${ }^{31 ; 39}$. Therefore, stresses in the bubble-caps of bubbles adjacent to collapsing cavities appear to be, at least, one order of magnitude higher than those observed around single collapsing cavities. Intuitively, this is finally not so surprising. Actually, after a bubble-cap's aperture, the now empty cavity has to collapse to 


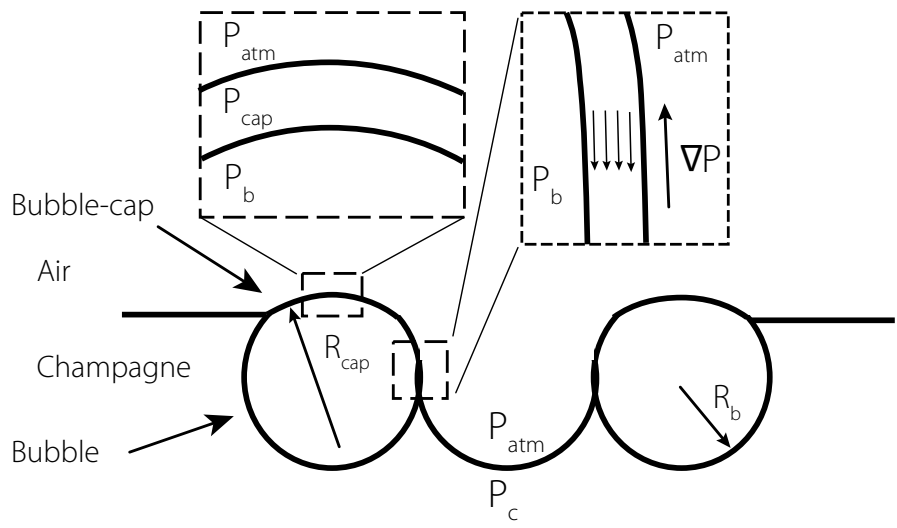

Fig. 26. Schematic transversal representation of the situation, as frozen after the disintegration of the central bubble-cap.

recover the horizontality of the liquid surface. The potential energy of such an unstable situation the difference of surface free energy between an hemispherical open cavity of radius $\mathrm{R}$ and a flat circular surface of radius $\mathrm{R}$ (of order of $\gamma \pi R^{2}$ ). Now, it should be noted that the same driving potential energy is responsible for the single cavity collapse and for the collapse of the cavity surrounded by neighboring bubble-caps. But, the volume of liquid displaced in the thin film of adjoining bubble-caps being much less than that of the boundary layer drawn, it logically induces higher energy dissipation rates per unit of volume and therefore higher strains in the "petals" of the flower-shaped structure. Finally, while absorbing the energy released during a bubble collapse, as so many tiny air-bags would do, adjoining bubble-caps store this energy into the thin liquid film of emerging bubble-caps, leading finally to stresses much higher than those observed in the boundary layer around single millimetric collapsing bubbles.

In addition to purely physicochemical reasons, biological reasons are also readily found for the investigation of such flower-shaped structures around bubbles collapsing in a bubble raft. Actually, in the biological industry, animal cells cultivated in bio-reactors were shown to be seriously damaged or even killed by the bursting of gas bubbles used to aerate the culture medium ${ }^{74 ; 75}$. It has even been suggested that structural deformations of adjacent tissues are induced by bubble collapse during laser-induced angioplasty ${ }^{76 ; 77}$. Kunas and Papoutsakis evaluated the critical shear stresses needed to cause irreversible damages to classical animal cells ${ }^{75}$. They found critical lethal stresses in the range between $10^{3}$ and $10^{4} \mathrm{~N} . \mathrm{m}^{-2}$. Therefore, by developing stresses of order of $10^{4}-10^{5} \mathrm{~N} . \mathrm{m}^{-2}$, bubbles bursting in a bubble raft should be potentially even more dangerous for micro-organisms or biological tissues trapped in the thin film of these "fast-stretched bubble-caps".

\subsection{Avalanches of bursting events in the bubble raft?}

Actually, avalanches of popping bubbles were put in evidence during the coarsening of bi-dimensional and three dimensional aqueous foams ${ }^{73 ; 78}$. How does the bubble raft behave at the surface of a flute poured with champagne? Does a bursting bubble produce a perturbation which extends to the neighboring bubbles and induce avalanches of bursting events which finally destroy the whole bubble raft? In case of champagne wines, a few time sequences of bubbles bursting in the bubble raft have been captured with a high-speed video camera. One of them is displayed in Fig. 27. 


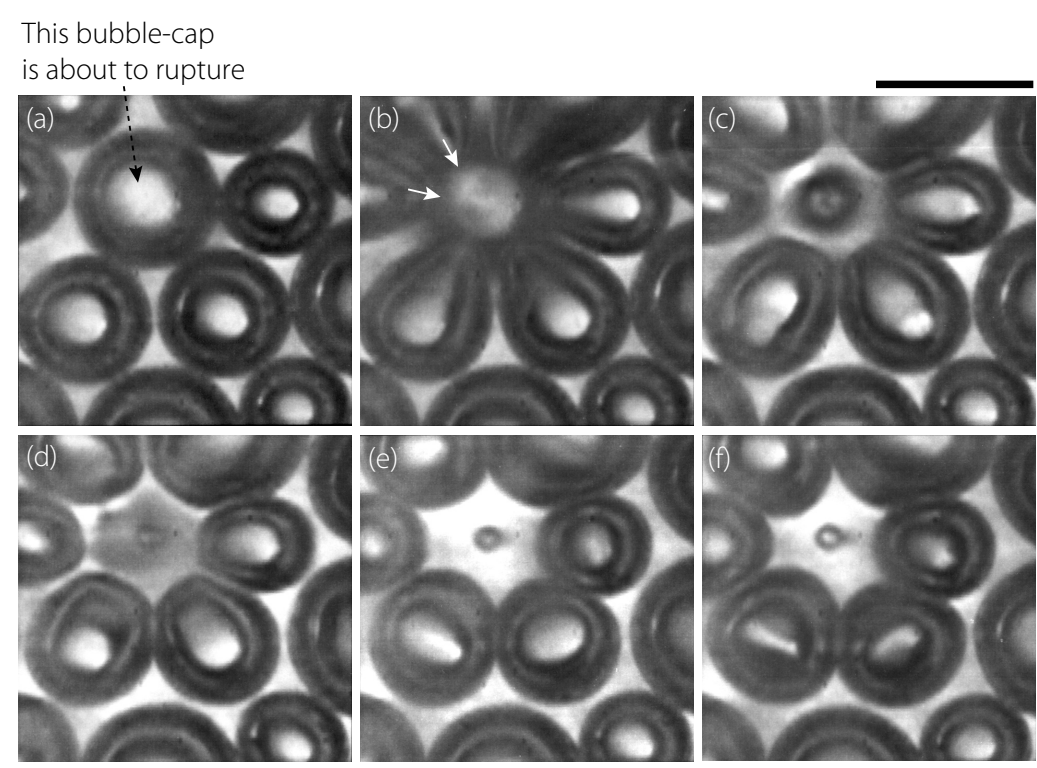

Fig. 27. Time sequence showing the dynamics of adjoining bubbles in touch with a collapsing one at the free surface of flute poured with champagne; the whole process was filmed at 1500 frames/s; from frame (d), in the center of the empty cavity left by the collapsing bubble, a tiny air-bubble entrapment is observed (bar $=1 \mathrm{~mm}$ ).

Between frame (a) and frame (b), the bubble pointed with the dashed arrow has disappeared. In frame (b), neighboring bubbles are literally sucked toward this now bubble-free area. Then, neighboring bubbles oscillate during a few milliseconds and progressively recover their initial hemispherical shape. In conclusion, in case of bubbles adjacent to collapsing ones, despite high shear stresses produced by a violent sucking process, bubbles adjacent to collapsing ones were never found to rupture and collapse in turn, thus causing a chain reaction. At the free surface of a flute poured with champagne, bursting events appear to be spatially and temporally non correlated. The absence of avalanches of bursting events seems to be linked to the champagne viscosity, which is about $50 \%$ higher than that of pure water ${ }^{73}$. It can also be noted that a tiny daughter bubble, approximately ten times smaller than the initial central bubble, has been entrapped during the collapsing process of the central cavity (as clearly seen in frames (c) and (d) of Fig. 27). Bubble entrapment during the collapsing process was already experimentally and numerically observed with single millimetric collapsing bubbles ${ }^{31 ; 79}$, including champagne bubbles ${ }^{26}$. This bubble entrapment process can also be observed as drops impact on liquid surfaces ${ }^{80 ; 81 ; 82 ; 83 ; 84}$.

\subsection{And finally what about the bubble bursting jet?}

Intuitively, differences between the dynamics of the jet from a single bubble and from a bubble bursting in a raft could be expected. Actually, the bulk shape of bubbles adjacent to the empty cavity left by the central collapsing bubble strongly changes the geometry of the system beneath the free surface. Such a situation could therefore modify the converging liquid flows all around the empty cavity, thus probably modifying in turn the overall dynamics of the upward liquid jet.

Even if the liquid jets shooting out from single collapsing bubbles are not always vertical, we expect bubble bursting jet from a bubble in a raft to be commonly tilted. 

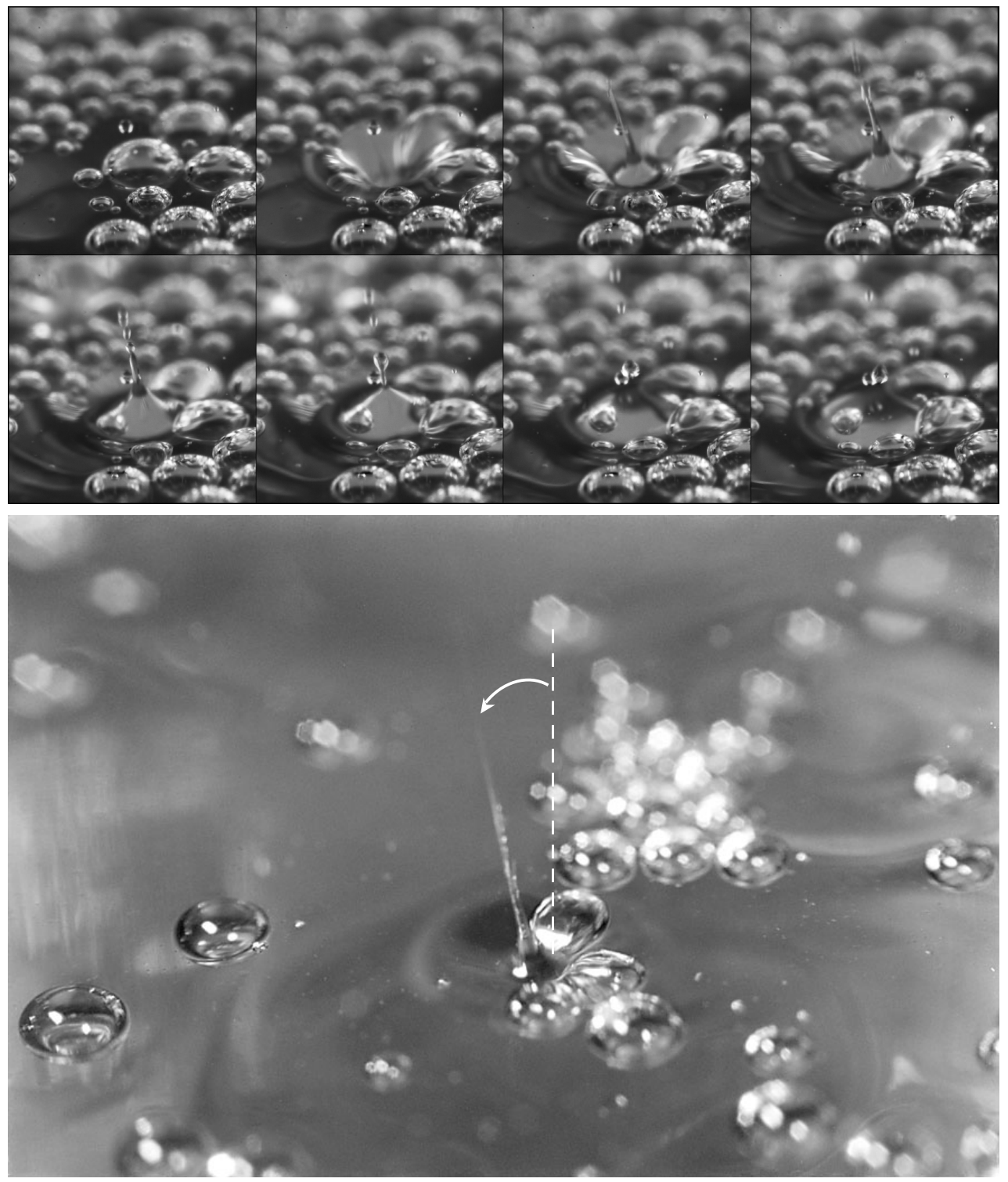

Fig. 28. Top images : Time sequence, filmed at 5000 frames per second showing formation of largely tilted jet as neighboring bubbles' symmetry is broken around collapsing bubble. Bottom image : Close-up on tilted jet as neighboring bubbles' symmetry is broken around collapsing one.

One example has been captured in the sequence of Fig. 28. How horizontal momentum is gained in these jets is still an unsettled question. We observe that this jet typically originates from bubbles located at the edge of a bubble raft. In this case, we expect that horizontal momentum lies in the initial shape of the collapsing bubble. Indeed, when isolated, the bubble is completely axisymmetric and so are the capillary waves riding on the bubble surface. The resulting jet preserves this axisymmetry and is vertical. Now when the bubble is located on the edge of the raft, there is a complete symmetry breaking in the initial shape. This strong asymmetry reflects in turn in the 
developing capillary waves, and eventually in the resulting tilted jet. More generally, we expect more asymmetry in raft bubble than in single bubble leading to more tilted jet. Such asymmetry-induced momentum is typical of, e.g. strong liquid jets produced during the collapse of cavitation bubble cloud and responsible for cavitation damage ${ }^{82}$.

The bottom image of Fig. 28 presents another example of tilted jet from a bubble at the edge of a raft ${ }^{85}$. indeed, on the right side of this picture, the collapsing bubble is bordered by three neighboring bubbles, whereas on the left side, there are no adjoining bubbles. The hexagonal symmetry is broken. The jet is tilted toward the "bubble-free" area. These are one example of the collective effects of bubble bursting in a raft but we expect others. For example, after the bubble rupture, a capillary wave is emitted and rides radially outwards. As the wave reaches distant bubbles, their rupture might occur on the wave, inducing most probably a tilted jet. The wave-induced horizontal momentum source is another form of asymmetry, and is reminiscent of the forward source of momentum of "walking droplets", rebounding on a vibrating liquid bath ${ }^{86}$.

Finally, the consequence of these collective effects on the drop size, velocity and in fine evaporation is not clear at all. Further experimental observations, combined with numerical simulations, are soon to be conducted in order to better understand the role of neighboring bubbles on the dynamics of the jet formation and its breakup into droplets.

\section{REFERENCES}

1. G. Liger-Belair, G. Polidori, and P. Jeandet, Chemical Society Reviews 37, 2490 (2008).

2. D. C. Blanchard and L. D. Syzdek, J. Geophys. Res. 93, 3649 (1988).

3. J. Wu, J. Phys. Oceanogr. 31, 3249 (2001).

4. H. Lhuissier and E. Villermaux, Journal of Fluid Mechanics 696, 5 (2012).

5. L. Duchemin, S. Popinet, C. Josserand, and S. Zaleski, Phys. Fluids 14, 3000 (2002).

6. J. S. Lee, B. M. Weon, S. J. Park, J. H. Je, K. Fezzaa, and W.-K. Lee, Nat Commun 2 (2011).

7. E. Ghabache, A. Antkowiak, C. Josserand, and T. Séon, Physics of Fluids (1994-present) 26, (2014).

8. D. C. Blanchard, Progress In Oceanography 1, 73 (1963).

9. D. E. Spiel, J. Geophys. Res. 99, 10289 (1994).

10. E. Ghabache and T. Séon, Phys. Rev. Fluids 1 (2016).

11. F. J. Resch, J. S. Darrozes, and G. M. Afeti, J. Geophys. Res. 91(C1), 1019 (1986).

12. A. H. Woodcock, C. F. Kientzler, A. B. Arons, and D. C. Blanchard, Nature 172, 1144 (1953).

13. F. MacIntyre, Journal of Geophysical Research 77, 5211 (1972).

14. J. Wu, Science 212, 324 (1981).

15. D. E. Spiel, J. Geophys. Res. 102, 5815 (1997).

16. E. R. Lewis and S. E. Schwartz, Sea Salt Aerosol Production. Mechanisms, Methods, Measurements, and Models, geophysical monograph 152. ed. (American Geophysical Union, Washington, DC, 2004).

17. C. D. O'Dowd and G. de Leeuw, Philosophical Transactions of the Royal Society A: Mathematical, Physical and Engineering Sciences 365, 1753 (2007).

18. C. D. O'Dowd, M. C. Facchini, F. Cavalli, D. Ceburnis, M. Mircea, S. Decesari, S. Fuzzi, Y. J. Yoon, and J.-P. Putaud, Nature 431, 676 (2004).

19. W. Barger and W. Garrett, Journal of Geophysical Research 75, 4561 (1970).

20. D. C. Blanchard, Tellus B 42, 200 (1990).

21. R. S. Tseng, J. T. Viechnicki, R. A. Skop, and J. W. Brown, Journal of Geophysical Research 97, 5201 (1992).

22. G. Liger-Belair, in Annales de physique, Vol. 31 (EDP sciences, 2006) pp. 1-133.

23. N. Péron, A. Cagna, M. Valade, C. Bliard, V. Aguié-Béghin, and R. Douillard, Langmuir 17, 791 (2001). 
24. N. Péron, J. Meunier, A. Cagna, M. Valade, and R. Douillard, Journal of microscopy 214, 89 (2004).

25. G. Liger-Belair, C. Cilindre, R. D. Gougeon, M. Lucio, I. Gebefügi, P. Jeandet, and P. Schmitt-Kopplin, Proc. Natl Acad. Sci. USA (2009).

26. G. Liger-Belair, H. Lemaresquier, B. Robillard, B. Duteurtre, and P. Jeandet, American journal of enology and viticulture 52, 88 (2001).

27. J. C. Bird, R. de Ruiter, L. Courbin, and H. A. Stone, Nature 465, 759 (2010).

28. J. Thomson, The London, Edinburgh, and Dublin Philosophical Magazine and Journal of Science 10, 330 (1855).

29. G. Liger-Belair and P. Jeandet, Langmuir 19, 801 (2003).

30. E. Ghabache, Surface libre hors équilibre : de l'effondrement de cavité aux jets étirés, Ph.D. thesis, UPMC (2015).

31. L. Duchemin, Quelques problèmes fortement non-linéaires de surface libre et leur résolution numérique, Ph.D. thesis, Université Aix-Marseille II (2001).

32. Y. TOBA, Journal of the Oceanographical Society of Japan 15, 121 (1959).

33. F. Culick, Journal of applied physics 31, 1128 (1960).

34. W. R. McEntee and K. J. Mysels, The Journal of Physical Chemistry 73, 3018 (1969).

35. A. Pandit and J. Davidson, Journal of Fluid Mechanics 212, 11 (1990).

36. J. Senée, B. Robillard, and M. Vignes-Adler, Food hydrocolloids 13, 15 (1999).

37. S. Hayami and Y. Toba, Journal of the Oceanographical Society of Japan 14, 145 (1958).

38. J. Wu, Journal of Geophysical Research 78, 511 (1973).

39. J. M. Boulton-Stone and J. R. Blake, Journal of Fluid Mechanics 254, 437 (1993).

40. D. E. Spiel, J. Geophys. Res. 100, 4995 (1995).

41. T. Séon and A. Antkowiak, Phys. Rev. Lett. 109 (2012).

42. B. W. Zeff, B. Kleber, J. Fineberg, and D. P. Lathrop, Nature 403, 401 (2000).

43. D. Bartolo, C. Josserand, and D. Bonn, Phys. Rev. Lett. 96, 124501 (2006).

44. J. B. Keller and M. J. Miksis, SIAM Journal on Applied Mathematics 43, 268 (1983).

45. F. H. Zhang and S. T. Thoroddsen, Physics of Fluids 20, 022104 (2008).

46. J. B. Keller, A. King, and L. Ting, Physics of Fluids (1994-present) 7, 226 (1995).

47. H. A. Stone and L. Leal, Journal of Fluid Mechanics 198, 399 (1989).

48. A. A. Castrejón-Pita, J. Castrejon-Pita, and I. Hutchings, Physical review letters 108, 074506 (2012).

49. M. Singh, H. M. Haverinen, P. Dhagat, and G. E. Jabbour, Advanced materials 22, $673(2010)$

50. J. M. Gordillo and S. Gekle, J. Fluid Mech. 663, 331 (2010).

51. E. Ghabache, T. Séon, and A. Antkowiak, J. Fluid Mech. 761, 206 (2014).

52. P. L. L. Walls, L. Henaux, and J. C. Bird, Phys. Rev. E 92, 021002 (2015).

53. C. F. Kientzler, A. B. Arons, D. C. Blanchard, and A. H. Woodcock, Tellus 6, 1 (1954).

54. S. R. Massel, Ocean Waves Breaking and Marine Aerosol Fluxes (Springer, 2007).

55. G. B. Deane and M. D. Stokes, Nature 418, 839 (2002).

56. L. Deike, W. K. Melville, and S. Popinet, Journal of Fluid Mechanics 801, 91 (2016).

57. G. Liger-Belair, A. Conreux, S. Villaume, and C. Cilindre, Food Research International 54, 516 (2013).

58. E. Ghabache, G. Liger-Belair, A. Antkowiak, and T. Séon, Scientific Reports 6, 25148 (2016).

59. D. Blanchard and A. Woodcock, Tellus 9, 145 (1957).

60. R. Clift, J. R. Grace, and M. E. Weber, Bubbles, drops, and particles, edited by D. Publications (Dover books on engineering, 2005).

61. L. Schiller and A. Naumann, Vdi Zeitung 77, 51 (1935).

62. A. Frohn and N. Roth, Dynamics of droplets (Springer Science \& Business Media, 2000).

63. W. A. Sirignano, Fluid dynamics and transport of droplets and sprays (Cambridge University Press, 1999).

64. A.-M. Cazabat and G. Guéna, Soft Matter 6, 2591 (2010).

65. I. Langmuir, Physical review 12, 368 (1918).

66. N. A. Fuchs, Evaporation and droplet growth in gaseous media, edited by P. Press (El- 
sevier, 1959).

67. W. Ranz and W. Marshall, Chem. Eng. Prog 48, 141 (1952).

68. E. Pohlhausen, ZAMM-Journal of Applied Mathematics and Mechanics/Zeitschrift für Angewandte Mathematik und Mechanik 1, 115 (1921).

69. F. Boulogne, A. Sauret, B. Soh, E. Dressaire, and H. A. Stone, Langmuir 31, 3094 (2015).

70. G. Liger-Belair, The Journal of Physical Chemistry B 118, 3156 (2014).

71. G. Liger-Belair, B. Robillard, M. Vignes-Adler, and P. Jeandet, Comptes Rendus de l'Académie des Sciences-Series IV-Physics 2, 775 (2001).

72. G. Liger-Belair and P. Jeandet, Langmuir 19, 5771 (2003).

73. H. Ritacco, F. Kiefer, and D. Langevin, Phys. Rev. Lett. 98, 244501 (2007).

74. A. Handa, A. Emery, and R. Spier, Developments in biological standardization 66, 241 (1986).

75. K. T. Kunas and E. T. Papoutsakis, Biotechnology and Bioengineering 36, 476 (1990).

76. T. Van Leeuwen, J. Meertens, E. Velema, M. Post, and C. Borst, Circulation 87, 1258 (1993).

77. E.-A. Brujan, EPL (Europhysics Letters) 50, 175 (2000).

78. N. Vandewalle, J. Lentz, S. Dorbolo, and F. Brisbois, Physical review letters 86, 179 (2001).

79. J. Herman and R. Mesler, Journal of Colloid and Interface Science 117, 565 (1987).

80. H. N. Oguz and A. Prosperetti, Journal of Fluid Mechanics 219, 143 (1990).

81. M. S. Longuet-Higgins, Journal of Fluid Mechanics 214, 395 (1990).

82. A. Prosperetti and H. N. Oguz, Annual Review of Fluid Mechanics 25, 577 (1993)

83. M. Rein, Journal of Fluid Mechanics 306, 145 (1996).

84. D. Morton, M. Rudman, and L. Jong-Leng, Physics of Fluids (1994-present) 12, 747 (2000).

85. G. Liger-Belair and G. Polidori, Voyage au coeur d'une bulle de champagne (Odile Jacob, 2011).

86. Y. Couder, S. Protière, E. Fort, and A. Boudaoud, Nature 437 (2005) 